\title{
LOCALIZED NONLINEAR FUNCTIONAL EQUATIONS AND TWO SAMPLING PROBLEMS IN SIGNAL PROCESSING
}

\author{
QIYU SUN
}

\begin{abstract}
Let $1 \leq p \leq \infty$. In this paper, we consider solving a nonlinear functional equation

$$
f(x)=y,
$$

where $x, y$ belong to $\ell^{p}$ and $f$ has continuous bounded gradient in an inverseclosed subalgebra of $\mathcal{B}\left(\ell^{2}\right)$, the Banach algebra of all bounded linear operators on the Hilbert space $\ell^{2}$. We introduce strict monotonicity property for functions $f$ on Banach spaces $\ell^{p}$ so that the above nonlinear functional equation is solvable and the solution $x$ depends continuously on the given data $y$ in $\ell^{p}$. We show that the Van-Cittert iteration converges in $\ell^{p}$ with exponential rate and hence it could be used to locate the true solution of the above nonlinear functional equation. We apply the above theory to handle two problems in signal processing: nonlinear sampling termed with instantaneous companding and subsequently average sampling; and local identification of innovation positions and qualification of amplitudes of signals with finite rate of innovation.
\end{abstract}

\section{INTRODUCTION}

Let $\ell^{p}:=\ell^{p}(\mathcal{N}), 1 \leq p \leq \infty$, be the space of all real-valued $p$-summable column vectors $x:=(x(n))_{n \in \mathcal{N}}$ on a countable index set $\mathcal{N}$ with its norm denoted by $\|\cdot\|_{p}:=$ $\|\cdot\|_{\ell^{p}(\mathcal{N})}$. In the first part of this paper, we consider solvability and stability of a nonlinear functional equation

$$
f(x)=y
$$

in Banach spaces $\ell^{p}, 1 \leq p \leq \infty$. We assume that both the original signal (image) $x$ to be recovered and the distorted signal (image) $y$ to be observed belong to $\ell^{p}, 1 \leq$ $p \leq \infty$. We also assume that the function $f$ in the nonlinear functional equation (1.1) has continuous bounded gradient $\nabla f$ in an inverse-closed Banach algebra of infinite matrices with certain off-diagonal decay (hence being localized and near sparse). Here the gradient $\nabla f$ of a function $f$ on $\ell^{p}$ is defined by

$$
\nabla f(x) c:=\lim _{t \rightarrow 0} \frac{f(x+t c)-f(x)}{t} \text { for } x, c \in \ell^{p} .
$$

Date: June 19, 2018.

1991 Mathematics Subject Classification. 47H07, 47J05, 65J15, 94A20, 94A12, 42C15, 46H99, 46T20, 47H05, 39B42.

Key words and phrases. Nonlinear functional equation, strict monotonicity, inverse-closedness, Banach algebra, infinite matrix, instantaneous companding, average sampling, signal with finite rate of innovation, shift-invariant space.

Research of the author was supported in part by the National Science Foundation (DMS1109063). 
Such a hypothesis is satisfied for functions $f$ modeling a distortion procedure that has strong neighborhood dependency; i.e., the change in amplitude of the distorted signal (image) $f(x)$ at each position depends essentially on the change in amplitudes of the original signal (image) $x$ at neighboring positions.

For the nonlinear functional equation (1.1) in the Hilbert space $\ell^{2}$, a well-known result is that it is solvable and stable provided that the function $f$ is strictly monotonic on $\ell^{2}$ and has continuous bounded gradient in $\mathcal{B}\left(\ell^{2}\right)$, see for instance [46, 69] or Lemma 2.3. Here $\mathcal{B}\left(\ell^{p}\right), 1 \leq p \leq \infty$, is the Banach algebra of all bounded linear operators on $\ell^{p}$, and a function $f$ on $\ell^{2}$ is said to be strictly monotonic [69] if there exists a positive constant $m_{0}$ such that

$$
\left(x-x^{\prime}\right)^{T}\left(f(x)-f\left(x^{\prime}\right)\right) \geq m_{0}\left(x-x^{\prime}\right)^{T}\left(x-x^{\prime}\right) \quad \text { for all } x, x^{\prime} \in \ell^{2} .
$$

The strict monotonicity constant of a function $f$ on $\ell^{2}$ is the largest constant $m_{0}$ such that (1.2) holds. If a function $f$ on $\ell^{2}$ has continuous gradient $\nabla f$ in $\mathcal{B}\left(\ell^{2}\right)$, an equivalent formulation of its strict monotonicity is that

$$
c^{T} \nabla f(x) c \geq m_{0} c^{T} c \quad \text { for all } c \in \ell^{2} \text { and } x \in \ell^{2} .
$$

In this paper, we consider the nonlinear functional equation (1.1) in Banach spaces $\ell^{p}, p \neq 2$, see Remark 5.4 and Section 8 for some of our motivations related to signal fidelity measures and local identification of innovations. We introduce the following "strict monotonicity" property for functions on $\ell^{p}, 1 \leq p \leq \infty$,

$$
c^{T} \nabla f(x) c \geq m_{0} c^{T} c \quad \text { for all } c \in \ell^{2} \text { and } x \in \ell^{p},
$$

where $m_{0}>0$. We call (1.4) the strict monotonicity property because of its similarity to the equivalent formulation (1.3) of the strict monotonicity for a function on $\ell^{2}$. We remark that the strict monotonicity property (1.4) for functions on Banach spaces could be verified more easily than the strong accretivity defined by Browder [9] and Kato [33] via normalized duality mapping, especially for functions arisen in nonlinear sampling and reconstruction. We show in Theorems 4.1 and 5.1 that the nonlinear functional equation (1.1) is solvable and stable in $\ell^{p}, 1 \leq p \leq \infty$, if $f$ has continuous bounded gradient in some Banach subalgebra of $\mathcal{B}\left(\ell^{2}\right)$ and satisfies the strict monotonicity property (1.4). The proofs of Theorems 4.1 and 5.1 rely heavily on a nonlinear version of Wiener's lemma (Theorem 2.1) and a unique Lipschitz extension theorem (Theorem 3.1) for strictly monotonic functions.

In the second part of this paper, we consider finding the true solution of the nonlinear functional equation (1.1) with $1 \leq p \leq \infty$. For a strictly monotonic function $f$ on $\ell^{2}$, consider the Van-Cittert iteration:

$$
x_{n+1}=x_{n}-\alpha\left(f\left(x_{n}\right)-y\right), \quad n \geq 0,
$$

where $x_{0} \in \ell^{2}$ is the initial guess and $\alpha>0$ is the relaxation factor. By the strict monotonicity property (1.3), we obtain that

$$
\left\|x_{n+1}-x_{n}\right\|_{2}^{2} \leq\left(1-2 \alpha m_{0}+\alpha^{2}\left(\sup _{x \in \ell^{2}}\|\nabla f(x)\|_{\mathcal{B}\left(\ell^{2}\right)}\right)\right)\left\|x_{n}-x_{n-1}\right\|_{2}^{2} \quad \text { for all } n \geq 1 .
$$


Thus we conclude that if $0<\alpha<2 m_{0} /\left(\sup _{x \in \ell^{2}}\|\nabla f(x)\|_{\mathcal{B}\left(\ell^{2}\right)}\right)$ then for any initial guess $x_{0}$, the sequence $x_{n}, n \geq 0$, in the Van-Cittert iteration (1.5) converges exponentially to the true solution $f^{-1}(y)$ of the nonlinear functional equation (1.1), i.e., there exist constants $C \in(0, \infty)$ and $r \in(0,1)$ such that

$$
\left\|x_{n}-f^{-1}(y)\right\|_{2} \leq C r^{n} \text { for all } n \geq 1 .
$$

In Theorem 6.1, we borrow a technique in [58, 60] used for establishing Wiener's lemma for infinite matrices, and we show that the sequence $x_{n}, n \geq 0$, in the VanCittert iteration method converges to the true solution $f^{-1}(y)$ in $\ell^{p}, 1 \leq p \leq \infty$, exponentially, provided that $0<\alpha<m_{0} /\left(\sup _{x \in \ell^{\infty}}\|\nabla f(x)\|_{\mathcal{B}\left(\ell^{2}\right)}+\sup _{x \in \ell^{\infty}}\|\nabla f(x)\|_{\mathcal{B}\left(\ell^{2}\right)}^{2}\right)$ and the function $f$ has continuous bounded gradient in some inverse-closed Banach algebra $\mathcal{B}$ satisfying the differential norm property (2.2). Therefore the VanCittert iteration method could be applied to locate the true solution of the (infinitedimensional) nonlinear functional equation (1.1) with $p \neq 2$. We are working on other methods that could solve the nonlinear functional equation (1.1) with $p \neq 2$ in a more stable and efficient way.

In the third part of this paper, we consider applying the above theory about solving the nonlinear functional equation (1.1) to two sampling problems in signal processing. We first apply it to a nonlinear sampling problem related to instantaneous companding

$$
F: h(t) \longmapsto F(h(t)),
$$

see Figure 1. The technique to transmit (process) the companded signal $F(h(t))$ instead of the original signal $h(t)$ is useful in a transmission system that does not respond well to very high or very low signal levels, since that instantaneous companding technique may amplify the weaker parts of a signal and de-emphasize its stronger parts. There are some theoretical obstacles in the path of instantaneous
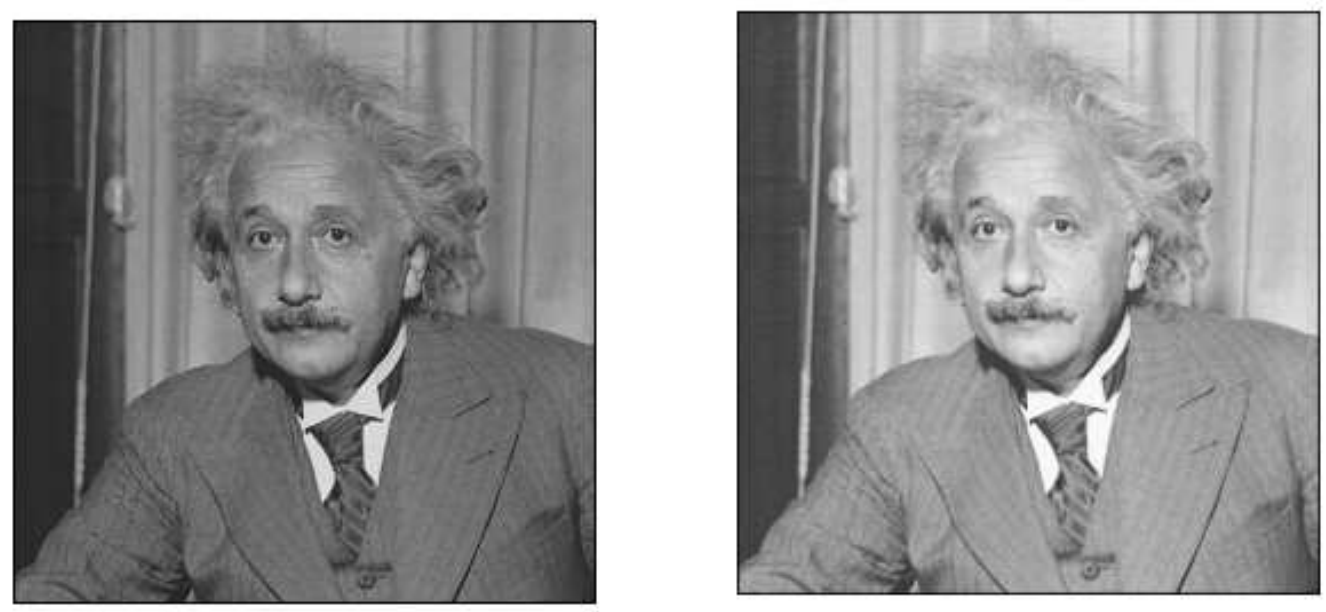

FiguRE 1. The left hand side is the original image, while the right hand side is the distorted image via $F(t)=255 \sin (\pi t / 510)$. 
companding. For instance, the instantaneous companding (1.6) does not preserve the bandlimitedness. A good news by Beurling [40] is that no information of a signal $h(t)$ bandlimited to $[-\pi \Omega, \pi \Omega], \Omega>0$, is lost by transmitting the companded signal $F(h(t))$ over bandlimiting channel $[-\pi \Omega, \pi \Omega]$, provided that the companding function $F$ is monotonic increasing. Precisely, the procedure of instantaneous companding and subsequently bandlimiting,

$$
B_{\pi \Omega} \ni h \stackrel{\text { instantaneous companding }}{\longrightarrow} F(h) \stackrel{\text { bandlimiting }}{\longrightarrow} P_{\pi \Omega}(F(h)) \in B_{\pi \Omega},
$$

is one-to-one on $B_{\pi \Omega}$ [40, 53]. Here $B_{\pi \Omega}$ is the Paley-Wiener space containing all square-integrable functions bandlimited to $[-\pi \Omega, \pi \Omega], P_{\pi \Omega}$ is the projection operator from $L^{2}$ to the Paley-Wiener space $B_{\pi \Omega}$, and $L^{p}:=L^{p}\left(\mathbb{R}^{d}\right), 1 \leq p \leq \infty$, is the space of all $p$-integrable functions on $\mathbb{R}^{d}$ with its norm denoted by $\|\cdot\|_{p}:=\|\cdot\|_{L^{p}\left(\mathbb{R}^{d}\right)}$, while the inner product on $L^{2}$ is denoted by $\langle\cdot, \cdot\rangle$.

In this paper, we consider a nonlinear sampling procedure of instantaneous companding and subsequently average sampling [17]:

$$
S_{F, \Psi}: h \stackrel{\text { instantaneous companding }}{\longrightarrow} F(h) \stackrel{\text { average sampling }}{\longrightarrow}\langle F(h), \Psi\rangle,
$$

where $\Psi=\left(\psi_{\gamma}\right)_{\gamma \in \Gamma}$ is the average sampler, $\Gamma \subset \mathbb{R}^{d}$ is the index set representing the location of the nonideal acquisition devices, and the sampling functional $\psi_{\gamma}$ at the sampling position $\gamma \in \Gamma$ reflects the characteristics of the acquisition device at that location. Such a nonlinear sampling procedure appears in a variety of setups and applications, such as charge-coupled device image sensors [31, 34, power electronics [51], radiometric photography, magnetic resonance imaging [18, 36, 54, and bandlimited signal transmission [40, 53. It would be an ill-posed problem to reconstruct a signal $h$ from its nonlinear sampling data $S_{F, \Psi}(h)$ if no a priori information on the original signal $h$ is given. In this paper, we assume that signals to be recovered are superposition of impulse response of varying positions and amplitudes,

$$
h(t)=\sum_{\lambda \in \Lambda} c(\lambda) \phi_{\lambda}(t)
$$

where $\Lambda$ is the index set with each $\lambda \in \Lambda$ representing an innovation position of the signal, $\Phi:=\left(\phi_{\lambda}\right)_{\lambda \in \Lambda}$ is the generator with each entry $\phi_{\lambda}$ being the impulse response of the signal generating device at the location $\lambda$, and $c:=(c(\lambda))_{\lambda \in \Lambda}$ is the amplitude vector with each entry $c(\lambda)$ being the amplitude of the signal at the innovation position $\lambda$, which is assumed to be $p$-summable for some $1 \leq p \leq \infty$ in this paper. In other words, signals to be recovered reside in the space

$$
V_{p}(\Phi):=\left\{c^{T} \Phi \mid c \in \ell^{p}(\Lambda)\right\}, 1 \leq p \leq \infty
$$

([61]). We remark that signals, such as bandlimited signals in a Paley-Wiener space, time signals in a finitely-generated shift-invariant space, time-frequency signals in Gabor analysis, and signals in a reproducing kernel subspace of $L^{p}$, have the parametric representation (1.8) [49, 59, 61]. 
Associated with the nonlinear sampling procedure (1.7) for signals in the space $V_{p}(\Phi)$ is the function

$f_{F, \Phi, \Psi}: \ell^{p}(\Lambda) \ni c \longrightarrow c^{T} \Phi \stackrel{\text { instantaneous companding and average sampling }}{\longrightarrow}\left\langle F\left(c^{T} \Phi\right), \Psi\right\rangle \in \ell^{p}(\Gamma)$.

For the function $f_{F, \Phi, \Psi}$ just defined, its gradient function

$$
\nabla f_{F, \Phi, \Psi}(x): \ell^{p}(\Lambda) \longmapsto \ell^{p}(\Gamma), x \in \ell^{p}(\Lambda)
$$

is a family of infinite matrices $\left(a_{\lambda, \gamma}(x)\right)_{\lambda \in \Lambda, \gamma \in \Gamma}$ with uniform off-diagonal decay, provided that the companding function $F$ has continuous bounded gradient and that both the generator $\Phi$ and the average sampler $\Psi$ are well-localized, such as when they have polynomial decay of order $\beta>d$, see Lemma 7.6. Here we say that $\Phi=\left(\phi_{\lambda}\right)_{\lambda \in \Lambda}$ has polynomial decay of order $\beta$ if

$$
\|\Phi\|_{\infty, \beta}:=\sup _{\lambda \in \Lambda}\left\|\phi_{\lambda}(\cdot)(1+|\cdot-\lambda|)^{\beta}\right\|_{\infty}<\infty .
$$

So we may apply the established solvability and stability of the nonlinear functional equation (1.1) to study the stability of the nonlinear sampling procedure (1.7), and the Van-Cittert iteration method to reconstruct signals $h \in V_{p}(\Phi)$ from its nonlinear sampling data $\langle F(h), \Psi\rangle$, see Theorems 7.1 and 7.7 for the theoretical results and Section 7.3 for the numerical simulations. The readers may refer to [17, 19] for the study of the nonlinear sampling procedure (1.7) from an engineering viewpoint.

A signal is said to have finite rate of innovation if it has finitely many degrees of freedom per unit of time [66]. In the second application of our theory about solving the nonlinear functional equation (1.1), we consider precise identification of innovation positions and accurate qualification of amplitudes of signals with finite rate of innovation. These are important to reach meaningful conclusions in many applications, such as global positioning system, ultra wide-band communication, and mass spectrometry. The readers may refer to [7, 16, 39, 42, 43, 45, 56, 66] and references therein for various techniques, such as the annihilating filter technique and the polynomial reproducing technique, that have been developed by Vetterli and his school to attack that challenging problem.

Signals living in the space $V_{p}(\Phi)$ have finite rate of innovation provided that the generator $\Phi=\left(\phi_{\lambda}\right)_{\lambda \in \Lambda}$ is well-localized (for instance, $\Phi$ has polynomial decay of order $\beta>d$ ) and the index set $\Lambda$ is a relatively-separated subset of $\mathbb{R}^{d}[61$. Here a subset $\Lambda$ of $\mathbb{R}^{d}$ is said to be relatively-separated if

$$
\sup _{t \in \mathbb{R}^{d}} \sum_{\lambda \in \Lambda} \chi_{\lambda+[0,1)^{d}}(t)<\infty
$$

where $\chi_{E}$ is a characteristic function on a measurable set $E$. In applications such as global positioning system, cellular radio, ultra wide-band communication, and mass spectrometry [11, 12, 15, 39], signals have the parametric representation (1.8) but both the innovation position set $\Lambda$ and the amplitude vector $c:=(c(\lambda))_{\lambda \in \Lambda}$ need to be determined. The dependence of the impulse response $\phi_{\lambda}$ at the innovation position $\lambda$ could be given or determined. For instance, the impulse response $\phi_{\lambda}$ is assumed to be approximately the shift of a given impulse $\varphi$ (i.e., $\phi_{\lambda} \approx \varphi(\cdot-\lambda)$ ) 
in [11, 12, 39], and the dilated shift of a given shape $\varphi$ with unknown width $w_{\lambda}$ (i.e., $\left.\phi_{\lambda} \approx \varphi\left((\cdot-\lambda) / w_{\lambda}\right)\right)$ in [15]. In this paper, we assume that the dependence of the impulse response $\phi_{\lambda}$ on the innovation position $\lambda$ is the shift of a given impulse response $\varphi$, i.e., $\phi_{\lambda}=\varphi(\cdot-\lambda)$. So signals have the following parametric representation

$$
h(t)=\sum_{\lambda \in \Lambda} c(\lambda) \varphi(\cdot-\lambda)
$$

We could also say that signals live in an almost-shift-invariant space generated by $\varphi$, as in the Fourier domain

$$
\hat{h}(\xi)=C(\xi) \hat{\phi}(\xi)
$$

is the product of an almost periodic function $C(\xi):=\sum_{\lambda \in \Lambda} c(\lambda) e^{-i \lambda \xi}$ and the Fourier transform $\hat{\varphi}$ of the impulse response $\varphi$. Here the Fourier transform $\hat{f}$ of an integrable function $f$ on $\mathbb{R}^{d}$ is defined by $\hat{f}(\xi)=\int_{\mathbb{R}^{d}} e^{-i x \xi} f(x) d x$.

In Section 8, we consider recovering a signal $h$ in the parametric representation (1.13) accurately from its nonlinear sampling data $\langle F(h), \Psi\rangle$, with a priori approximate information on innovation positions and amplitudes. For that purpose, we require that the linearization of the nonlinear sampling process

$$
\left(\begin{array}{l}
\Lambda \\
c
\end{array}\right) \text { parametric representation } \stackrel{(1.13)}{\longrightarrow} h \stackrel{\text { companding and sampling }}{\longrightarrow}\langle F(h), \Psi\rangle
$$

is stable at the given approximate innovation position vector $\Lambda_{0}$ and amplitude vector $c_{0}=\left(c_{0}(\lambda)\right)_{\lambda \in \Lambda_{0}}$; i.e., there exist positive constants $A$ and $B$ such that

$$
A\|e\|_{2} \leq\left\|S_{\Lambda_{0}, c_{0}} e\right\|_{2} \leq B\|e\|_{2} \text { for all } e \in\left(\ell^{2}\left(\Lambda_{0}\right)\right)^{d+1},
$$

where

$$
S_{\Lambda_{0}, c_{0}}:=\left(\begin{array}{c}
-c_{0}(\lambda)\left\langle F^{\prime}\left(h_{0}\right) \nabla \varphi(\cdot-\lambda), \psi_{\gamma}\right\rangle \\
\left\langle F^{\prime}\left(h_{0}\right) \varphi(\cdot-\lambda), \psi_{\gamma}\right\rangle
\end{array}\right)_{\gamma \in \Gamma, \lambda \in \Lambda_{0}}
$$

and $h_{0}=\sum_{\lambda \in \Lambda_{0}} c_{0}(\lambda) \varphi(\cdot-\lambda)$. Associated with the linearization of the nonlinear sampling process (1.15) at $\left(\Lambda_{0}, c_{0}\right)$ is the function

$$
f_{\Lambda_{0}, c_{0}, \Psi}:\left(\begin{array}{c}
\sigma \\
c
\end{array}\right) \longmapsto\left(S_{\Lambda_{0}, c_{0}}^{T} S_{\Lambda_{0}, c_{0}}\right)^{-1} S_{\Lambda_{0}, c_{0}}^{T}\left\langle F(h)-F\left(h_{0}\right), \Psi\right\rangle,
$$

where $\sigma=(\sigma(\lambda))_{\lambda \in \Lambda_{0}}, c=(c(\lambda))_{\lambda \in \Lambda_{0}}$ and $h=\sum_{\lambda \in \Lambda_{0}}\left(c_{0}(\lambda)+c(\lambda)\right) \varphi(\cdot-\lambda-\sigma(\lambda))$. We observe that the gradient of the function $f_{\Lambda_{0}, c_{0}, \Psi}$ is a family of infinite matrices with certain off-diagonal decay, and that $f_{\Lambda_{0}, c_{0}, \Psi}$ has strict monotonicity property in a small neighborhood of the origin; i.e.,

$$
\inf _{\|e\|_{\left(\ell^{2}\left(\Lambda_{0}\right)\right)^{d+1}}=1} e^{T} \nabla f_{\Lambda_{0}, c_{0}, \Psi}\left(\begin{array}{c}
\sigma \\
c
\end{array}\right) e>0
$$

for all $\sigma \in\left(\ell^{\infty}\left(\Lambda_{0}\right)\right)^{d}$ and $c \in \ell^{\infty}\left(\Lambda_{0}\right)$ with $\|c\|_{\infty}+\|\sigma\|_{\infty} \leq \delta_{0}$, where $\delta_{0}>0$ is a sufficiently small number. Thus we may apply our theory about solving the nonlinear functional equation (1.1) with $p=\infty$ indirectly to local identification of innovation positions and qualification of amplitudes, see Theorem 8.1 . 
Finally we consider the following natural questions: 1) how to find approximate innovation positions and amplitudes from the given sampling data and 2) how to verify the stability condition (1.16) for the linearization matrix. From the stability condition (1.16) it follows that approximate innovation positions should be separated from each other and that approximate amplitudes at innovation positions should be above certain level. So we may model those signals as superposition of impulse response of active and nonactive generating devices located at an unknown neighborhood of a uniform grid; i.e., after appropriate scaling, we may assume that signals live in a perturbed shift-invariant space

$$
V_{\infty, \oslash}(\varphi ; \sigma):=\left\{\sum_{k \in \mathbb{Z}^{d}} c(k) \varphi(\cdot-k-\sigma(k)) \mid(c(k))_{k \in \mathbb{Z}^{d}} \in \ell_{\oslash}^{\infty}\left(\mathbb{Z}^{d}\right)\right\}
$$

with unknown perturbation $\sigma:=(\sigma(k))_{k \in \mathbb{Z}^{d}}$, where

$$
\ell_{\oslash}^{\infty}\left(\mathbb{Z}^{d}\right)=\left\{c:=\left.(c(k))_{k \in \mathbb{Z}^{d}}\left|\|c\|_{\ell_{\varnothing}^{\infty}}:=\sup _{c(k) \neq 0}\right| c(k)|+| c(k)\right|^{-1}<\infty\right\} .
$$

We may assume that signals live in the infinite unions of almost-shift-invariant spaces $\cup_{\sigma} V_{\infty, \oslash}(\varphi ; \sigma)$, see [41] for sampling in finite unions of subspaces. A negative result for sampling in the perturbed shift-invariant space with unknown perturbations is that not all signals in such a space can be recovered from their samples provided that $\varphi$ satisfies the popular and traditional Strang-Fix condition. The reason is that in this case, one cannot determine the perturbation $\sigma_{0}$ of signals $\sum_{k \in \mathbb{Z}^{d}} \varphi\left(\cdot-k-\sigma_{0}\right), \sigma_{0} \in$ $\mathbb{R}^{d}$, as they have same constant amplitudes and are identical. In Theorem 8.3 , we provide a positive result for sampling in a perturbed shift-invariant space with unknown perturbations. We show that any signal $h$ in a perturbed shift-invariant space $V_{\infty, \oslash}(\varphi ; \sigma)$ with small perturbation error $\|\sigma\|_{\infty}$ can be recovered from its sampling data $\left\{\left\langle h, \psi_{m}(\cdot-k)\right\rangle \mid 1 \leq m \leq M, k \in \mathbb{Z}^{d}\right\}$ provided that

$$
\operatorname{rank}\left(\begin{array}{ccc}
{\left[\widehat{\nabla \varphi}, \widehat{\psi}_{1}\right](\xi)} & \cdots & {\left[\widehat{\nabla \varphi}, \widehat{\psi}_{M}\right](\xi)} \\
{\left[\hat{\varphi}, \widehat{\psi}_{1}\right](\xi)} & \cdots & {\left[\hat{\varphi}, \widehat{\psi}_{M}\right](\xi)}
\end{array}\right)=d+1 \quad \text { for all } \xi \in[-\pi, \pi]^{d},
$$

see Remark 8.4 for the connection of the above assumption with sampling in shiftinvariant spaces. Here the bracket product $[f, g]$ of two square-integrable functions $f$ and $g$ is given by $[f, g](\xi)=\sum_{l \in \mathbb{Z}^{d}} f(\xi+2 l \pi) \overline{g(\xi+2 l \pi)}$.

The paper is organized as follows. The first part of this paper contains four sections concerning solvability and stability of the nonlinear functional equation (1.1). The starting point of solvability is Wiener's lemma for strictly monotone functions in Section 2. A sufficient condition for the solvability of the nonlinear functional equation (1.1) in $\ell^{p}$ is introduced in Section 4, while the unique Lipschitz extension theorem in Section 3 and Wiener's lemma in Section 2 are crucial in the proof. The stability of the nonlinear functional equation (1.1) in $\ell^{p}$ is studied in Section 5. The central pieces of the second part of this paper are the global exponential convergence of the Van-Cittert iteration in $\ell^{p}$ and the local R-quadratic convergence of the quasi-Newton iteration in $\ell^{p}, 1 \leq p \leq \infty$, see Section 6 for details. Our proof of the global exponential convergence depends heavily on the paracompactness idea used 
in [58, 60] to establish Wiener's lemma for infinite matrices. In the third part of this paper, we apply solvability and stability of the nonlinear functional equation (1.1) in the first part and numerical implementation in the second part to two sampling problems in signal processing: 1) stable recovery of signals $h$ living in the space $V_{p}(\Phi)$ from their nonlinear sampling data $\langle F(h), \Psi\rangle$ in Sections 7 , and 2) local identification of innovation positions and qualification of amplitudes of signals $h$ having the parametric representation (1.13) from their nonlinear sampling data $\langle F(h), \Psi\rangle$ in Section 8.

In this paper, the capital letter $C$ denotes an absolute constant, which may be different at different occurrences.

\section{Nonlinear Wiener's LEMma}

A Banach subalgebra $\mathcal{A}$ of $\mathcal{B}$ is said to be inverse-closed if an element in $\mathcal{A}$, that is invertible in $\mathcal{B}$, is also invertible in $\mathcal{A}$. Inverse-closedness occurs in many fields of mathematics under various names, such as spectral invariance, Wiener pair, and local subalgebra. Inverse-closedness (= Wiener's lemma) has been established for infinite matrices satisfying various off-diagonal decay conditions (or equivalently for localized linear functions on $\ell^{p}$ ), see for instance [5, 6, 20, 23, 26, 32, 55, 57, 58, 60, 63. and the survey papers [21, 37]. Wiener's lemma for infinite matrices plays crucial roles for well-localization of dual wavelet frames and Gabor frames [4, 20, 32, 38, algebra of pseudo-differential operators [22, 29, 57, fast numerical implementation [10, 13, 28, stable signal recovery [1, 7, 28, 30, 59, 61, and optimization [47, 48].

A quantitative version of inverse-closedness is to admit norm control, which is fundamental in our study of the nonlinear functional equation (1.1) and its applications to sampling in signal processing. Here an inverse-closed Banach subalgebra $\mathcal{A}$ of $\mathcal{B}$ is said to admit norm control in $\mathcal{B}$ if there exists a continuous function $h$ from $[0, \infty) \times[0, \infty)$ to $[0, \infty)$ such that

$$
\left\|A^{-1}\right\|_{\mathcal{A}} \leq h\left(\|A\|_{\mathcal{A}},\left\|A^{-1}\right\|_{\mathcal{B}}\right)
$$

for all $A \in \mathcal{A}$ with $A^{-1} \in \mathcal{B}$. Unlike the inverse-closedness, there are not many papers devoted to the above quantitative version of inverse-closedness [23, 24, 50, 64]. The algebra $\mathcal{W}$ of commutative infinite matrices of the form $A:=(a(i-j))_{i, j \in \mathbb{Z}}$ with norm $\|A\|_{\mathcal{W}}=\sum_{j \in \mathbb{Z}}|a(j)|$ is inverse-closed in $\mathcal{B}\left(\ell^{2}\right)$ by the classical Wiener's lemma [68] but it does not admit norm control [50]. The $C^{*}$-subalgebras $\mathcal{A}$ of $\mathcal{B}$ with a common unit and a differential norm, i.e.,

$$
\|A B\|_{\mathcal{A}} \leq C\left(\|A\|_{\mathcal{A}}\|B\|_{\mathcal{B}}+\|A\|_{\mathcal{B}}\|B\|_{\mathcal{A}}\right) \text { for all } A, B \in \mathcal{A} \subset \mathcal{B}
$$

was shown in [23] to admit norm control. The smoothness described in the above differential subalgebra $\mathcal{A}$ of $\mathcal{B}$ has been widely used in operator theory and noncommutative geometry [8, 35, 52] and also appears in approximation theory [14]. For the quantitative version of inverse-closedness, we will show in Proposition 2.4 that a Banach subalgebra $\mathcal{A}$ of $\mathcal{B}\left(\ell^{2}\right)$ admits norm control in $\mathcal{B}\left(\ell^{2}\right)$ if it contains the identity matrix $I$, it is closed under the transpose operation, and it satisfies

$\|A B\|_{\mathcal{A}} \leq C_{0}\left(\|A\|_{\mathcal{A}}\|B\|_{\mathcal{A}}^{\theta}\|B\|_{\mathcal{B}\left(\ell^{2}\right)}^{1-\theta}+\|B\|_{\mathcal{A}}\|A\|_{\mathcal{A}}^{\theta}\|A\|_{\mathcal{B}\left(\ell^{2}\right)}^{1-\theta}\right) \quad$ for all $A, B \in \mathcal{A} \subset \mathcal{B}\left(\ell^{2}\right)$, 
where $C_{0} \in(0, \infty)$ and $\theta \in[0,1)$. The above inequality (2.2), a weak form of the differential norm property (2.1), is satisfied by many families of Banach subalgebras of $\mathcal{B}\left(\ell^{2}\right)$ [26, 32, 58, 60, 63, and it will also be used later to guarantee the exponential convergence of the Van-Cittert iteration method, see Theorem 6.1.

The main topic of this section is to introduce Wiener's lemma for strictly monotonic functions, see Theorem 2.1. It states that a strictly monotonic function on $\ell^{2}$ with continuous bounded gradient in some inverse-closed subalgebra of $\mathcal{B}\left(\ell^{2}\right)$ has its inverse with continuous bounded gradient in the same Banach subalgebra. The above nonlinear Wiener's lemma will be used later to establish the unique extension theorem (Theorem 3.1) and the invertibility theorem (Theorem 4.1) for functions with continuous bounded gradient in a Banach subalgebra of $\mathcal{B}\left(\ell^{2}\right)$.

Theorem 2.1. Let $\mathcal{A}$ be a Banach subalgebra of $\mathcal{B}\left(\ell^{2}\right)$ that admits norm control in $\mathcal{B}\left(\ell^{2}\right)$. If $f$ is a strictly monotonic function on $\ell^{2}$ such that its gradient $\nabla f$ is bounded and continuous in $\mathcal{A}$ (i.e., $\sup _{x \in \ell^{2}}\|\nabla f(x)\|_{\mathcal{A}}<\infty$ and $\lim _{x^{\prime} \rightarrow x}$ in $\ell^{2} \| \nabla f\left(x^{\prime}\right)-$ $\nabla f(x) \|_{\mathcal{A}}=0$ for all $\left.x \in \ell^{2}\right)$, then $f$ is invertible and its inverse $f^{-1}$ has continuous bounded gradient $\nabla\left(f^{-1}\right)$ in $\mathcal{A}$.

For a relatively-separated subset $\Lambda$ in (1.12) and a nonnegative number $\beta \geq 0$, define the Jaffard class $\mathcal{J}_{\beta}(\Lambda)$ of infinite matrices $A=\left(a\left(\lambda, \lambda^{\prime}\right)\right)_{\lambda, \lambda^{\prime} \in \Lambda}$ by

$$
\mathcal{J}_{\beta}(\Lambda):=\left\{A\left|\|A\|_{\mathcal{J}_{\beta}(\Lambda)}:=\sup _{\lambda, \lambda^{\prime} \in \Lambda}\left(1+\left|\lambda-\lambda^{\prime}\right|\right)^{\beta}\right| a\left(\lambda, \lambda^{\prime}\right) \mid<\infty\right\}
$$

[32, 58. The Jaffard class can be interpreted as the set of infinite matrices having polynomial off-diagonal decay of degree $\beta$. It has been established in [58, 60] that the Jaffard class $\mathcal{J}_{\beta}(\Lambda)$ in (2.3) with $\beta>d$ is closed under the transpose operation, it contains the identity matrix $I$, and it satisfies the differential norm property (2.2). Hence the Jaffard class $\mathcal{J}_{\beta}(\Lambda)$ with $\beta>d$ admits norm control in $\mathcal{B}\left(\ell^{2}\right)$ by Proposition 2.4 at the end of this section. Then applying Theorem 2.1 to the Banach algebra $\mathcal{J}_{\beta}(\Lambda)$ leads to the following conclusion.

Corollary 2.2. Let $\beta>d$ and $\Lambda$ be a relatively-separated subset of $\mathbb{R}^{d}$. If $f$ is a strictly monotonic function on $\ell^{2}(\Lambda)$ such that its gradient $\nabla f$ is bounded and continuous in $\mathcal{J}_{\beta}(\Lambda)$, then $f$ is invertible and its inverse $f^{-1}$ has continuous bounded gradient in $\mathcal{J}_{\beta}(\Lambda)$.

To prove Theorem 2.1, we recall a textbook version, i.e., $\mathcal{A}=\mathcal{B}\left(\ell^{2}\right)$, about invertibility of a strictly monotonic function on $\ell^{2}$, see for instance [69].

Lemma 2.3. Let $f$ be a strictly monotonic function on $\ell^{2}$ such that its gradient $\nabla f$ is bounded and continuous in $\mathcal{B}\left(\ell^{2}\right)$. Then $f$ is invertible and its inverse $f^{-1}$ has continuous bounded gradient in $\mathcal{B}\left(\ell^{2}\right)$.

Proof of Theorem 2.1. Since $\mathcal{A}$ is a Banach subalgebra of $\mathcal{B}\left(\ell^{2}\right)$, the gradient $\nabla f$ is also bounded and continuous in $\mathcal{B}\left(\ell^{2}\right)$ by the assumptions on $f$. Then $f$ is invertible and the gradient of its inverse $f^{-1}$ is bounded and continuous in $\mathcal{B}\left(\ell^{2}\right)$ by Lemma 2.3. Therefore the gradient $\nabla\left(f^{-1}\right)$ is bounded in $\mathcal{A}$ as $\mathcal{A}$ admits norm control in 
$\mathcal{B}\left(\ell^{2}\right)$ and

$$
\nabla f\left(f^{-1}(x)\right) \nabla f^{-1}(x)=I
$$

by $f\left(f^{-1}(x)\right)=x$ for all $x \in \ell^{2}$. Combining (2.4) with the boundedness of $\nabla f$ in $\mathcal{A}$ and the continuity of $f^{-1}$ on $\ell^{2}$, we obtain that for any $y_{0} \in \ell^{2}$

$$
\begin{aligned}
\left\|\nabla f^{-1}(y)-\nabla f^{-1}\left(y_{0}\right)\right\|_{\mathcal{A}} & =\left\|\left(\nabla f\left(f^{-1}(y)\right)\right)^{-1}-\left(\nabla f\left(f^{-1}\left(y_{0}\right)\right)\right)^{-1}\right\|_{\mathcal{A}} \\
& \leq\left(\sup _{z \in \ell^{2}}\left\|(\nabla f(z))^{-1}\right\|_{\mathcal{A}}\right)^{2}\left\|\nabla f\left(f^{-1}(y)\right)-\nabla f\left(f^{-1}\left(y_{0}\right)\right)\right\|_{\mathcal{A}} \\
& \rightarrow 0 \text { as } y \rightarrow y_{0} \text { in } \ell^{2} .
\end{aligned}
$$

This proves the continuity of $\nabla\left(f^{-1}\right)$ in $\mathcal{A}$.

Denote by $A^{T}$ the transpose of an infinite matrix $A$. We conclude this section by showing that a Banach subalgebra $\mathcal{A}$ of $\mathcal{B}\left(\ell^{2}\right)$ satisfying (2.5), a weak version of the differential norm property (2.2), admits norm control in $\mathcal{B}\left(\ell^{2}\right)$.

Proposition 2.4. Let $\mathcal{A}$ be a Banach subalgebra of $\mathcal{B}\left(\ell^{2}\right)$ such that it contains the identity matrix I and it is closed under the transpose operation. If there exist positive constants $D \in(0, \infty)$ and $\theta \in[0,1)$ such that

$$
\left\|A^{2}\right\|_{\mathcal{A}} \leq D\|A\|_{\mathcal{A}}^{1+\theta}\|A\|_{\mathcal{B}\left(\ell^{2}\right)}^{1-\theta} \quad \text { for all } A \in \mathcal{A}
$$

then $\mathcal{A}$ is an inverse-closed Banach subalgebra of $\mathcal{B}\left(\ell^{2}\right)$. Moreover, for any $A \in \mathcal{A}$ with $A^{-1} \in \mathcal{B}\left(\ell^{2}\right)$,

$$
\left\|A^{-1}\right\|_{\mathcal{A}} \leq\left\|A^{T}\right\|_{\mathcal{A}}\|A\|_{\mathcal{B}\left(\ell^{2}\right)}^{-2} \sum_{n=0}^{\infty} r^{n}\left(D\left(\|I\|_{\mathcal{A}}+\left\|A^{T}\right\|_{\mathcal{A}}\|A\|_{\mathcal{A}}\left\|A^{-1}\right\|_{\mathcal{B}\left(\ell^{2}\right)}^{-2}\right) r^{-1}\right)^{\frac{1+\theta}{\theta} n^{\log _{2}(1+\theta)}}
$$

if $\theta \in(0,1)$, and

$$
\left\|A^{-1}\right\|_{\mathcal{A}} \leq\left\|A^{T}\right\|_{\mathcal{A}}\|A\|_{\mathcal{B}\left(\ell^{2}\right)}^{-2} \sum_{n=0}^{\infty} r^{n}\left(D\left(\|I\|_{\mathcal{A}}+\left\|A^{T}\right\|_{\mathcal{A}}\|A\|_{\mathcal{A}}\left\|A^{-1}\right\|_{\mathcal{B}\left(\ell^{2}\right)}^{-2}\right) r^{-1}\right)^{1+\log _{2} n}
$$

if $\theta=0$, where $r=1-\left(\left\|A^{-1}\right\|_{\mathcal{B}\left(\ell^{2}\right)}\|A\|_{\mathcal{B}\left(\ell^{2}\right)}\right)^{-2} \in[0,1)$.

Proof. We provide only a brief sketch of the proof as we can mimic the argument in [58] where $\mathcal{A}$ is the Jaffard class. Let $A \in \mathcal{A}$ with $A^{-1} \in \mathcal{B}\left(\ell^{2}\right)$. Then the product $A^{T} A$ between $A$ and its transpose $A^{T}$ is a positive operator on $\ell^{2}$ by the invertibility assumption on $A$,

$$
(1-r) I \leq \frac{A^{T} A}{\|A\|_{\mathcal{B}\left(\ell^{2}\right)}^{2}} \leq I
$$

for some positive constant $r=1-\left(\left\|A^{-1}\right\|_{\mathcal{B}\left(\ell^{2}\right)}\|A\|_{\mathcal{B}\left(\ell^{2}\right)}\right)^{-2} \in[0,1)$. Set $B=I-$ $A^{T} A /\|A\|_{\mathcal{B}\left(\ell^{2}\right)}^{2}$. Then

$$
\|B\|_{\mathcal{A}} \leq\|I\|_{\mathcal{A}}+\left\|A^{T}\right\|_{\mathcal{A}}\|A\|_{\mathcal{A}}\|A\|_{\mathcal{B}\left(\ell^{2}\right)}^{-2}<\infty
$$


as $\mathcal{A}$ is a Banach algebra closed under the transpose operation. Using (2.5) and (2.8), and recalling that $\mathcal{A}$ is a Banach algebra, we obtain that for all $n \geq 0$,

$$
\left\|B^{2 n+1}\right\|_{\mathcal{A}} \leq\|B\|_{\mathcal{A}}\left\|B^{2 n}\right\|_{\mathcal{A}}
$$

and

$$
\left\|B^{2 n}\right\|_{\mathcal{A}} \leq D\left\|B^{n}\right\|_{\mathcal{B}\left(\ell^{2}\right)}^{1-\theta}\left\|B^{n}\right\|_{\mathcal{A}}^{1+\theta} \leq C_{0} r^{n(1-\theta)}\left\|B^{n}\right\|_{\mathcal{A}}^{1+\theta}
$$

where $D$ and $\theta$ are given in (2.5). Applying (2.10) and (2.11) iteratively, we get

$$
\begin{aligned}
\left\|B^{n}\right\|_{\mathcal{A}} & \leq D\|B\|_{\mathcal{A}}^{\epsilon_{0}} r^{\frac{1-\theta}{2} \sum_{i=1}^{k} \epsilon_{i} 2^{i}}\left\|B^{\sum_{i=1}^{k} \epsilon_{i} 2^{i-1}}\right\|_{\mathcal{A}}^{1+\theta} \leq \cdots \\
& \leq D^{\sum_{j=0}^{k-1}(1+\theta)^{j}}\|B\|_{\mathcal{A}}^{\sum_{j=0}^{k-1} \epsilon_{j}(1+\theta)^{j}} r^{\frac{1-\theta}{2} \sum_{j=1}^{k}\left(\sum_{i=j}^{k} \epsilon_{i} 2^{i}\right)\left(\frac{1+\theta}{2}\right)^{j-1}}\left\|B^{\epsilon_{k}}\right\|_{\mathcal{A}}^{(1+\theta)^{k}} \\
12) \leq\left(D\|B\|_{\mathcal{A}} r^{-1}\right)^{\frac{1+\theta}{\theta} n^{\log _{2}(1+\theta)}} r^{n} & \leq
\end{aligned}
$$

if $\theta \in(0,1)$, and

$$
\left\|B^{n}\right\|_{\mathcal{A}} \leq\left(D\|B\|_{\mathcal{A}} r^{-1}\right)^{1+\log _{2} n} r^{n}
$$

if $\theta=0$, where $n=\sum_{i=0}^{k} \epsilon_{i} 2^{i}$ with $\epsilon_{i} \in\{0,1\}$ and $\epsilon_{k}=1$. Then $A^{-1} \in \mathcal{A}$ as

$$
A^{-1}=\left(A^{T} A\right)^{-1} A^{T}=\|A\|_{\mathcal{B}\left(\ell^{2}\right)}^{-2} \sum_{n=0}^{\infty} B^{n} A^{T} .
$$

The estimates (2.6) and (2.7) hold by (2.9), (2.12), (2.13) and (2.14).

\section{UNIQUE EXTENSION}

In this section, we apply the nonlinear Wiener's lemma (Theorem 2.1) established in the previous section, and show that a strictly monotonic function $f$ on $\ell^{2}$ with continuous bounded gradient in some Banach algebra can be uniquely extended to a continuously invertible function on $\ell^{p}, 1 \leq p \leq \infty$.

Theorem 3.1. Let $\mathcal{N}$ be a countable index set, $\mathcal{A}$ be a Banach subalgebra of $\mathcal{B}\left(\ell^{2}(\mathcal{N})\right)$ that admits norm control in $\mathcal{B}\left(\ell^{2}(\mathcal{N})\right)$, and let $f$ be a strictly monotonic function on $\ell^{2}(\mathcal{N})$ with the property that $f(0)=0$ and the gradient $\nabla f$ is bounded and continuous in $\mathcal{A}$. Then

(i) the function $f$ can be uniquely extended to a continuous function $F$ on $\ell^{p}(\mathcal{N}), 1 \leq p<\infty$, if $\mathcal{A}$ is a Banach subalgebra of $\mathcal{B}\left(\ell^{p}(\mathcal{N})\right)$; and

(ii) the function $f$ can be uniquely extended to a continuous function $F$ on $\ell^{\infty}(\mathcal{N})$ with respect to the weak-star topology, provided that $\mathcal{A}$ is a Banach subalgebra of $\mathcal{B}\left(\ell^{\infty}(\mathcal{N})\right)$ and that there is a family of nested finite subsets $\mathcal{N}_{m}, m \geq 1$, of $\mathcal{N}$ such that $\cup_{m=1}^{\infty} \mathcal{N}_{m}=\mathcal{N}, \mathcal{N}_{m} \subset \mathcal{N}_{m+1}$ for all $m \geq 1$, and the projection operators $P_{m}, m \geq 1$, defined by

$$
P_{m} x=\left(x(n) \chi_{\mathcal{N}_{m}}(n)\right)_{n \in \mathcal{N}} \text { for } x=(x(n))_{n \in \mathcal{N}} \in \ell^{\infty}(\mathcal{N}),
$$

satisfy

$$
\lim _{m \rightarrow \infty} \sup _{\|A\|_{\mathcal{A}} \leq 1}\left\|P_{m} A\left(I-P_{m+1}\right)\right\|_{\mathcal{B}\left(\ell^{\infty}(\mathcal{N})\right)}=0
$$


Moreover, the above unique extension $F$ has continuous inverse $F^{-1}$ on $\ell^{p}(\mathcal{N}), 1 \leq$ $p \leq \infty$, and satisfies the following uniform Lipschitz conditions:

$$
\left\|F(x)-F\left(x^{\prime}\right)\right\|_{p} \leq\left(\sup _{z \in \ell^{2}(\mathcal{N})}\|\nabla f(z)\|_{\mathcal{A}}\right)\left(\sup _{\|A\|_{\mathcal{A}} \leq 1}\|A\|_{\mathcal{B}\left(\ell^{p}(\mathcal{N})\right)}\right)\left\|x-x^{\prime}\right\|_{p}
$$

and

$$
\left\|F^{-1}(x)-F^{-1}\left(x^{\prime}\right)\right\|_{p} \leq\left(\sup _{z \in \ell^{2}(\mathcal{N})}\left\|\nabla f^{-1}(z)\right\|_{\mathcal{A}}\right)\left(\sup _{\|A\|_{\mathcal{A}} \leq 1}\|A\|_{\mathcal{B}\left(\ell^{p}(\mathcal{N})\right)}\right)\left\|x-x^{\prime}\right\|_{p}
$$

for all $x, x^{\prime} \in \ell^{p}(\mathcal{N}), 1 \leq p \leq \infty$.

The technical assumption (3.1) for a Banach algebra $\mathcal{A}$ of $\mathcal{B}\left(\ell^{\infty}(\mathcal{N})\right)$ can be thought as a weak version of band-limited approximability, as it holds if

$$
\lim _{m \rightarrow \infty} \sup _{\|A\|_{\mathcal{A}} \leq 1}\left\|A-A_{m}\right\|_{\mathcal{B}\left(\ell^{\infty}(\mathcal{N})\right)}=0
$$

where $A_{m}=\left(a(i, j) \chi_{\rho(i, j) \leq K}\right)_{i, j \in \mathcal{N}}$ is the band-limited truncation of $A=(a(i, j))_{i, j \in \mathcal{N}} \in$ $\mathcal{A}$, and $\rho$ is a distance function on $\mathcal{N} \times \mathcal{N}$ with $\sup _{i \in \mathcal{N}} \#\{j \in \mathcal{N}: \rho(i, j) \leq m\}<\infty$ for all $m \geq 1$. We remark that (3.1) is invalid for the classical Schur class

$$
\mathcal{S}:=\left\{(a(i, j))_{i, j \in \mathbb{Z}}: \sup _{i \in \mathbb{Z}} \sum_{j \in \mathbb{Z}}|a(i, j)|+\sup _{j \in \mathbb{Z}} \sum_{i \in \mathbb{Z}}|a(i, j)|<\infty\right\} .
$$

Given $\beta>d$ and a relatively-separated subset $\Lambda$ of $\mathbb{R}^{d}$, we notice that the Jaffard class $\mathcal{J}_{\beta}(\Lambda)$ is a Banach subalgebra of $\mathcal{B}\left(\ell^{p}(\Lambda)\right)$ for all $1 \leq p \leq \infty$, and also that (3.1) holds by letting $\mathcal{N}_{m}=\left\{\lambda \in \Lambda:|\lambda| \leq 2^{m}\right\}, m \geq 1$. Therefore a strictly monotonic function $f$ on $\ell^{2}(\Lambda)$ with continuous bounded gradient in the Jaffard class $\mathcal{J}_{\beta}(\Lambda)$ can be extended to a continuously invertible function on $\ell^{p}(\Lambda), 1 \leq p \leq \infty$.

Corollary 3.2. Let $\beta>d, \Lambda$ be a relatively-separated subset of $\mathbb{R}^{d}$, and let $f$ be a strictly monotonic function on $\ell^{2}(\Lambda)$ such that $f(0)=0$ and the gradient $\nabla f$ is bounded and continuous in $\mathcal{J}_{\beta}(\Lambda)$. Then the function $f$ can be extended to a continuously invertible function on $\ell^{p}(\Lambda), 1 \leq p \leq \infty$.

To prove Theorem 3.1, we need a technical lemma about the extension of a function on $\ell^{q}, 1 \leq q<\infty$, which is not necessarily strictly monotonic.

Lemma 3.3. Let $1 \leq q<\infty, \mathcal{N}$ be a countable index set, $\mathcal{A}$ be a Banach subalgebra of $\mathcal{B}\left(\ell^{q}(\mathcal{N})\right)$, and $f$ be a continuous function on $\ell^{q}(\mathcal{N})$ such that $f(0)=0$ and its gradient $\nabla f$ is bounded and continuous in $\mathcal{A}$. Then

(i) the function $f$ can be uniquely extended to a continuous function $F$ on $\ell^{p}(\mathcal{N})$ if $1 \leq p<\infty$ and $\mathcal{A}$ is a Banach subalgebra of $\mathcal{B}\left(\ell^{p}(\mathcal{N})\right)$; and

(ii) the function $f$ can be uniquely extended to a continuous function $F$ on $\ell^{\infty}(\mathcal{N})$ with respect to the weak-star topology if $\mathcal{A}$ is a Banach subalgebra of $\mathcal{B}\left(\ell^{\infty}(\mathcal{N})\right)$ and satisfies (3.1).

Furthermore the extension F satisfies the uniform Lipschitz condition (3.2). 
Proof. (i) Given $x \in \ell^{p}:=\ell^{p}(\mathcal{N}), 1 \leq p<\infty$, take a Cauchy sequence $x_{n} \in$ $\ell^{p} \cap \ell^{q}, n \geq 1$, that converges to $x$ in $\ell^{p}$ and satisfies $x_{1}=0$. For any $x^{\prime}, x^{\prime \prime} \in \ell^{p} \cap \ell^{q}$,

$$
\begin{aligned}
\left\|f\left(x^{\prime}\right)-f\left(x^{\prime \prime}\right)\right\|_{p} & =\left\|\left(\int_{0}^{1} \nabla f\left(s x^{\prime}+(1-s) x^{\prime \prime}\right) d s\right)\left(x^{\prime}-x^{\prime \prime}\right)\right\|_{p} \\
& \leq\left(\sup _{0 \leq s \leq 1}\left\|\nabla f\left(s x^{\prime}+(1-s) x^{\prime \prime}\right)\right\|_{\mathcal{B}\left(\ell^{p}\right)}\right)\left\|x^{\prime}-x^{\prime \prime}\right\|_{p} \\
& \leq\left(\sup _{z \in \ell^{q}}\|\nabla f(z)\|_{\mathcal{A}}\right)\left(\sup _{\|A\|_{\mathcal{A}} \leq 1}\|A\|_{\mathcal{B}\left(\ell^{p}\right)}\right)\left\|x^{\prime}-x^{\prime \prime}\right\|_{p} .
\end{aligned}
$$

Thus $f\left(x_{n}\right), n \geq 1$, is a Cauchy sequence in $\ell^{p}$. Denote the limit of $f\left(x_{n}\right), n \geq 1$, by $F(x)$; i.e., $F(x):=\lim _{n \rightarrow \infty} f\left(x_{n}\right)$ in $\ell^{p}$. This together with (3.4) implies that the function $F$ on $\ell^{p}$ is a continuous extension of the function $f$ on $\ell^{q}$ and satisfies the uniform Lipschitz condition (3.2). Furthermore such a continuous extension is unique due to the density of $\ell^{p} \cap \ell^{q}$ in $\ell^{p}$.

(ii) Let $P_{m}, m \geq 1$, be as in Theorem 3.1 . For every $m \geq 1$,

$$
\begin{aligned}
\left\|P_{m} f(x)\right\|_{\infty} & \leq\|f(x)-f(0)\|_{\infty} \leq \int_{0}^{1}\|\nabla f(s x) x\|_{\infty} d s \\
& \leq\left(\sup _{z \in \ell^{q}}\|\nabla f(z)\|_{\mathcal{A}}\right)\left(\sup _{\|A\|_{\mathcal{A}} \leq 1}\|A\|_{\mathcal{B}\left(\ell^{\infty}\right)}\right)\|x\|_{\infty}
\end{aligned}
$$

for $x \in \ell^{q}$, and

$$
\begin{aligned}
& \left\|P_{m} f(x)-P_{m} f\left(x^{\prime}\right)\right\|_{\infty} \\
= & \left\|\int_{0}^{1} P_{m} \nabla f\left(s x+(1-s) x^{\prime}\right)\left(I-P_{k}\right)\left(x-x^{\prime}\right) d s\right\|_{\infty} \\
\leq & \left(\sup _{z \in \ell^{q}}\|\nabla f(z)\|_{\mathcal{A}}\right)\left(\sup _{\|A\|_{\mathcal{A}} \leq 1}\left\|P_{m} A\left(I-P_{k}\right)\right\|_{\mathcal{B}\left(\ell^{\infty}\right)}\right)\left\|x-x^{\prime}\right\|_{\infty}
\end{aligned}
$$

for all $x, x^{\prime} \in \ell^{q}$ with $P_{k} x=P_{k} x^{\prime}$. The above two estimates together with (3.1) imply that $P_{m} f\left(P_{n} x\right), n \geq 1$, is a Cauchy sequence in $\ell^{\infty}$ for every $x \in \ell^{\infty}$ and $m \geq 1$. Denote the limit of the above Cauchy sequence by $F_{m}(x)$. Clearly $P_{m^{\prime}} F_{m}(x)=$ $F_{m^{\prime}}(x)$ for all sequences $x \in \ell^{\infty}$ and integers $m^{\prime}$ and $m$ with $1 \leq m^{\prime} \leq m$. This implies that there is a unique sequence, to be denoted by $F(x)$, such that

$$
P_{m} F(x)=F_{m}(x) \text { for all } x \in \ell^{\infty} \text { and } m \geq 1 .
$$

The function $F$ is well-defined on $\ell^{\infty}$ with $F(0)=0$ because

$$
\|F(x)\|_{\infty}=\sup _{m \geq 1} \lim _{n \rightarrow \infty}\left\|P_{m} f\left(P_{n} x\right)\right\|_{\infty} \leq\left(\sup _{z \in \ell^{q}}\|\nabla f(z)\|_{\mathcal{A}}\right)\left(\sup _{\|A\|_{\mathcal{A}} \leq 1}\|A\|_{\mathcal{B}\left(\ell^{\infty}\right)}\right)\|x\|_{\infty}
$$

by (3.5). Moreover, by (3.5), (3.6) and (3.7),

$$
\left\|F(x)-F\left(x^{\prime}\right)\right\|_{\infty} \leq\left(\sup _{z \in \ell^{q}}\|\nabla f(z)\|_{\mathcal{A}}\right)\left(\sup _{\|A\|_{\mathcal{A}} \leq 1}\|A\|_{\mathcal{B}\left(\ell^{\infty}\right)}\right)\left\|x-x^{\prime}\right\|_{\infty}
$$


for all $x, x^{\prime} \in \ell^{\infty}$; and

$$
\begin{aligned}
\left\|P_{m} F(x)-P_{m} F\left(P_{n} x\right)\right\|_{\infty} \leq & \left(\sup _{\|A\|_{\mathcal{A}} \leq 1}\left\|P_{n-1} A\left(I-P_{n}\right)\right\|_{\mathcal{B}\left(\ell^{\infty}\right)}\right) \\
& \times\left(\sup _{z \in \ell^{q}}\|\nabla f(z)\|_{\mathcal{A}}\right)\|x\|_{\infty}
\end{aligned}
$$

for all $x \in \ell^{\infty}$ and $m \leq n-1$. Denote by $\langle\cdot, \cdot\rangle$ the action between sequences in $\ell^{1}$ and $\ell^{\infty}$. From (3.8), (3.9) and (3.10) it follows that

$$
\begin{aligned}
& \left|\langle y, F(x)\rangle-\left\langle y, F\left(x^{\prime}\right)\right\rangle\right| \\
\leq \quad & \left\|\left(I-P_{m}\right) y\right\|_{1}\left(\|F(x)\|_{\infty}+\left\|F\left(x^{\prime}\right)\right\|_{\infty}\right) \\
+ & \left\|P_{m} y\right\|_{1}\left(\left\|P_{m} F(x)-P_{m} F\left(P_{m+1} x\right)\right\|_{\infty}\right. \\
& \left.+\left\|P_{m} F\left(x^{\prime}\right)-P_{m} F\left(P_{m+1} x^{\prime}\right)\right\|_{\infty}\right) \\
& +\left\|P_{m} y\right\|_{1}\left\|P_{m} F\left(P_{m+1} x\right)-P_{m} F\left(P_{m+1} x^{\prime}\right)\right\|_{\infty} \\
\leq \quad & \left(\sup _{z \in \ell^{q}}\|\nabla f(z)\|_{\mathcal{A}}\right)\left(\|y\|_{1} \sup _{\|A\|_{\mathcal{A}} \leq 1}\left\|P_{m} A\left(I-P_{m+1}\right)\right\|_{\mathcal{B}\left(\ell^{\infty}\right)}\right. \\
& \left.+\left\|\left(I-P_{m}\right) y\right\|_{1} \sup _{\|A\|_{\mathcal{A}} \leq 1}\|A\|_{\mathcal{B}\left(\ell^{\infty}\right)}\right)\left(\|x\|_{\infty}+\left\|x^{\prime}\right\|_{\infty}\right) \\
& +\left(\sup _{z \in \ell^{q}}\|\nabla f(z)\|_{\mathcal{A}}\right)\left(\sup _{\|A\|_{\mathcal{A}} \leq 1}\|A\|_{\mathcal{B}\left(\ell^{\infty}\right)}\right)\|y\|_{1}\left\|P_{m+1}\left(x-x^{\prime}\right)\right\|_{\infty}
\end{aligned}
$$

for all $y \in \ell^{1}, x, x^{\prime} \in \ell^{\infty}$ and $m \geq 1$. This together with the off-diagonal decay assumption (3.1) proves the continuity of the function $F$ with respect to the weakstar topology. Thus $F$ is an extension of the function $f$ on $\ell^{q}$ which is continuous with respect to the weak-star topology and satisfies the uniform Lipschitz condition (3.2). The uniqueness of such an extension follows from the fact that $\ell^{\infty} \cap \ell^{q}$ is dense in $\ell^{\infty}$ with respect to the weak-star topology.

Proof of Theorem 3.1. Let $F$ be the unique extension of the function $f$ to $\ell^{p}$ in Lemma 3.3, Then $F$ satisfies (3.2) by Lemma 3.3. By Theorem 2.1, $\nabla f^{-1}$ is continuous and bounded in $\mathcal{A}$. Applying Lemma 3.3 to the function $f^{-1}$ on $\ell^{2}$ leads to the unique extension $G$ of the function $f^{-1}$ to $\ell^{p}$. By Lemma 3.3. $G$ is the continuous inverse of the function $F$ on $\ell^{p}$ that satisfies (3.3), because $\ell^{p} \cap \ell^{2}$ is dense in $\ell^{p}$ with respect to the strong topology if $1 \leq p<\infty$ and the weak-star topology of $\ell^{\infty}$ if $p=\infty$.

\section{INVERTIBILITY}

In this section, we apply the unique Lipschitz extension theorem (Theorem 3.1) established in the previous section, and show that the restriction of a function $f$ on $\ell^{\infty}$ to $\ell^{p} \subset \ell^{\infty}, 1 \leq p \leq \infty$, is invertible on $\ell^{p}$ if its gradient $\nabla f$ is bounded and continuous in some Banach algebra and has the strict monotonicity property (4.1).

Theorem 4.1. Let $2 \leq p_{0} \leq \infty$, $\mathcal{A}$ be a subalgebra of $\mathcal{B}\left(\ell^{q}\right)$ for all $1 \leq q \leq \infty$ with the property that it admits norm control in $\mathcal{B}\left(\ell^{2}\right)$ and satisfies (3.1). Assume that $f$ 
is a continuous function on $\ell^{p_{0}}$ such that $f(0)=0$, its gradient $\nabla f$ is bounded and continuous in $\mathcal{A}$ and satisfies

$$
c^{T} \nabla f(x) c \geq m_{0} c^{T} c \quad \text { for all } c \in \ell^{2} \text { and } x \in \ell^{p_{0}}
$$

where $m_{0}>0$. Then

(i) for every $1 \leq p \leq p_{0}$, the restriction of $f$ to $\ell^{p}$ is a continuously invertible function on $\ell^{p}$ and the gradient of its inverse is bounded and continuous in $\mathcal{A}$; and

(ii) for every $p_{0}<p \leq \infty$, the function $f$ can be extended to a continuously invertible function $F$ on $\ell^{p}$ that satisfies

$$
\left\|F(x)-F\left(x^{\prime}\right)\right\|_{p} \leq\left(\sup _{z \in \ell^{p_{0}}}\|\nabla f(z)\|_{\mathcal{A}}\right)\left(\sup _{\|A\|_{\mathcal{A}} \leq 1}\|A\|_{\mathcal{B}\left(\ell^{p}\right)}\right)\left\|x-x^{\prime}\right\|_{p}
$$

and

$$
\left\|F^{-1}(x)-F^{-1}\left(x^{\prime}\right)\right\|_{p} \leq\left(\sup _{z \in \ell^{p} 0}\left\|\nabla f^{-1}(z)\right\|_{\mathcal{A}}\right)\left(\sup _{\|A\|_{\mathcal{A}} \leq 1}\|A\|_{\mathcal{B}\left(\ell^{p}\right)}\right)\left\|x-x^{\prime}\right\|_{p}
$$

for all $x, x^{\prime} \in \ell^{p}$.

We do not know whether or not the conclusions in the above invertibility theorem hold when $1 \leq p_{0}<2$. Taking $p=p_{0}$ in Theorem 4.1 leads to the following generalization of the well known result for invertibility of a function on $\ell^{2}$, see [46, 69] or Lemma 2.3.

Corollary 4.2. Let $2 \leq p \leq \infty$, and $\mathcal{A}$ be a subalgebra of $\mathcal{B}\left(\ell^{q}\right)$ for all $1 \leq q \leq \infty$ with the property that it admits norm control in $\mathcal{B}\left(\ell^{2}\right)$ and satisfies (3.1). If $f$ is a continuous function on $\ell^{p}$ such that $f(0)=0$, its gradient $\nabla f$ is bounded and continuous in $\mathcal{A}$ and satisfies

$$
c^{T} \nabla f(x) c \geq m_{0} c^{T} c \quad \text { for all } c \in \ell^{2} \text { and } x \in \ell^{p},
$$

then $f$ has continuous inverse in $\ell^{p}$ and the inverse $f^{-1}$ has continuous bounded gradient in $\mathcal{A}$.

Remark 4.3. Applying Corollary 4.2 to a linear function $f(x)=A x$, where $A$ is an infinite matrix in $\mathcal{A}$, we conclude that if $A$ is positive definite, i.e., $C_{1} I \leq A \leq C_{2} I$ for some positive constants $C_{1}$ and $C_{2}$, then $A$ has bounded inverse in $\mathcal{B}\left(\ell^{p}\right)$ for all $1 \leq p \leq \infty$. The above result follows from Wiener's lemma for infinite matrices in $\mathcal{A}$ if we assume further that $\mathcal{A}$ is closed under transpose operation and has the identity $I$ as its unit [6, 21, 23, 24, 26, 37, 55, 60, 63].

Taking the Jaffard class $\mathcal{J}_{\beta}(\Lambda)$ as the Banach algebra $\mathcal{A}$ in Theorem4.1, we obtain

Corollary 4.4. Let $\beta>d, \Lambda$ be a relatively-separated subset of $\mathbb{R}^{d}$, and let $f$ be a continuous function on $\ell^{\infty}(\Lambda)$ such that $f(0)=0$, and its gradient $\nabla f$ is bounded and continuous in $\mathcal{J}_{\beta}(\Lambda)$ and satisfies (4.1). Then for every $1 \leq p \leq \infty$, the restriction of $f$ to $\ell^{p}(\Lambda)$ is a continuously invertible function on $\ell^{p}(\Lambda)$, and the gradient of its inverse is bounded and continuous in $\mathcal{J}_{\beta}(\Lambda)$. 
Proof of Theorem 4.1. (i) First we prove that for every $1 \leq p \leq p_{0}$ the restriction of the function $f$ on $\ell^{p}$, denoted by $F_{p}$, is well-defined. Let $P_{m}, m \geq 1$, be as in Theorem 3.1. Take $x \in \ell^{p} \subset \ell^{p_{0}}$, then $P_{m} f(x) \in \ell^{p}$ and

$$
\begin{aligned}
\left\|P_{m} f(x)\right\|_{p} & =\left\|P_{m} f(x)-P_{m} f(0)\right\|_{p} \leq \int_{0}^{1}\left\|P_{m} \nabla f(s x) x\right\|_{p} d s \\
& \leq\left(\sup _{z \in \ell^{p_{0}}}\|\nabla f(z)\|_{\mathcal{B}\left(\ell^{p}\right)}\right)\|x\|_{p} \quad \text { for all } m \geq 1,
\end{aligned}
$$

which implies that

$$
\|f(x)\|_{p}=\sup _{m \geq 1}\left\|P_{m} f(x)\right\|_{p} \leq\left(\sup _{z \in \ell^{p} 0}\|\nabla f(z)\|_{\mathcal{A}}\right)\left(\sup _{\|A\|_{\mathcal{A}} \leq 1}\|A\|_{\mathcal{B}\left(\ell^{p}\right)}\right)\|x\|_{p}
$$

for all $x \in \ell^{p}$. Thus the restriction of $f$ on $\ell^{p}$ is well-defined.

Next we prove that for every $1 \leq p \leq p_{0}$, the function $F_{p}$ is continuous on $\ell^{p}$ and has continuous bounded gradient in $\mathcal{A}$. Similar to the argument in (4.4), we obtain

$$
\left\|F_{p}\left(x^{\prime}\right)-F_{p}(x)\right\|_{p} \leq\left(\sup _{z \in \ell^{p_{0}}}\|\nabla f(z)\|_{\mathcal{A}}\right)\left(\sup _{\|A\|_{\mathcal{A}} \leq 1}\|A\|_{\mathcal{B}\left(\ell^{p}\right)}\right)\left\|x-x^{\prime}\right\|_{p}
$$

and

$$
\begin{aligned}
\| F_{p}\left(x^{\prime}\right)- & F_{p}(x)-\nabla f(x)\left(x^{\prime}-x\right) \|_{p} \leq\left(\sup _{\|A\|_{\mathcal{A}} \leq 1}\|A\|_{\mathcal{B}\left(\ell^{p}\right)}\right) \\
& \times\left(\sup _{0 \leq s \leq 1}\left\|\nabla f\left(x+s\left(x^{\prime}-x\right)\right)-\nabla f(x)\right\|_{\mathcal{A}}\right)\left\|x-x^{\prime}\right\|_{p}
\end{aligned}
$$

for all $x, x^{\prime} \in \ell^{p}$. This implies that $F_{p}$ is continuous on $\ell^{p}$ and

$$
\nabla F_{p}(x)=\nabla f(x) \text { for all } 1 \leq p \leq p_{0}
$$

Hence $F_{p}$ has its gradient $\nabla F_{p}(x)$ in $\mathcal{A}$. The continuity of $\nabla F_{p}(x), x \in \ell^{p}$, in $\mathcal{A}$ then follows from the continuity of the gradient $\nabla f(x), x \in \ell^{p_{0}}$, in $\mathcal{A}$ and the continuous embedding of $\ell^{p}$ in $\ell^{p_{0}}$ for every $1 \leq p \leq p_{0}$.

Then we prove the continuous invertibility of the function $F_{p}$ on $\ell^{p}, 1 \leq p \leq$ $p_{0}$. We notice that the function $F_{2}$ satisfies all requirements for the function $f$ in Theorem 3.1 because it has continuous gradient in $\mathcal{A}$ and strictly monotonic property by (4.1), (4.5) and the assumption $p_{0} \geq 2$, and it satisfies $F_{2}(0)=0$ by the assumption $f(0)=0$. Thus by Theorem 3.1 it suffices to show that the function $F_{p}$ is a continuous extension of the function $F_{2}$ on $\ell^{p}$ with respect to the strong topology when $1 \leq p<\infty$ and the weak-star topology when $p=\infty$. The above extension property follows as both $F_{2}$ and $F_{p}$ are the restriction of the function $f$ on $\ell^{p_{0}}$. The continuity of the function $F_{p}$ with respect to the strong topology when $1 \leq p<\infty$ follows as $F_{p}$ is continuous on $\ell^{p}$. To establish the continuity of the function $F_{p}$ with respect to the weak-star topology when $p=\infty$, we notice that $p_{0}=\infty$ and $F_{p}=f$ in this case. Moreover for $p=\infty$, similar to the argument used to establish (3.11) 
we obtain

$$
\begin{aligned}
& \left|\left\langle y, F_{p}(x)\right\rangle-\left\langle y, F_{p}\left(x^{\prime}\right)\right\rangle\right| \\
\leq \quad & \left(\sup _{z \in \ell^{\infty}}\|\nabla f(z)\|_{\mathcal{A}}\right)\left(\|y\|_{1} \sup _{\|A\|_{\mathcal{A}} \leq 1}\left\|P_{m} A\left(I-P_{m+1}\right)\right\|_{\mathcal{B}\left(\ell^{\infty}\right)}\right. \\
& \left.+\left\|\left(I-P_{m}\right) y\right\|_{1} \sup _{\|A\|_{\mathcal{A}} \leq 1}\|A\|_{\mathcal{B}\left(\ell^{\infty}\right)}\right)\left(\|x\|_{\infty}+\left\|x^{\prime}\right\|_{\infty}\right) \\
& +\left(\sup _{z \in \ell^{\infty}}\|\nabla f(z)\|_{\mathcal{A}}\right)\left(\sup _{\|A\|_{\mathcal{A}} \leq 1}\|A\|_{\mathcal{B}(\ell \infty)}\right)\|y\|_{1}\left\|P_{m+1}\left(x-x^{\prime}\right)\right\|_{\infty}
\end{aligned}
$$

for all $y \in \ell^{1}, x, x^{\prime} \in \ell^{\infty}$ and $m \geq 1$, which implies that $F_{p}$ is continuous on $\ell^{\infty}$ with respect to the weak-star topology.

Finally we prove that for every $1 \leq p \leq p_{0}$, the inverse of the function $F_{p}$ has its gradient being bounded and continuous in $\mathcal{A}$. Take $x \in \ell^{p}$, and let $x_{n} \in \ell^{p} \cap \ell^{2}, n \geq 1$, converge to $x$ with respect to the strong topology of $\ell^{p}$ when $1 \leq p<\infty$ and the weak-star topology of $\ell^{\infty}$ when $p=\infty$. Recall that the function $F_{p}$ is a continuous extension of the function $F_{2}$ on $\ell^{p}$ with respect to the strong topology when $1 \leq p<$ $\infty$ and the weak-star topology when $p=\infty$. Then

$$
\lim _{n \rightarrow \infty} c^{T}\left(F_{2}\left(x_{n}+t c\right)-F_{2}\left(x_{n}\right)\right)=c^{T}\left(F_{p}(x+t c)-F_{p}(x)\right),
$$

where $t \in \mathbb{R}$ and $c \in \ell^{0}$ (the space of all finitely supported sequences). This together with (4.1) and (4.5) implies that

$$
\begin{aligned}
c^{T} \nabla F_{p}(x) c & =\lim _{t \rightarrow 0} t^{-1} c^{T}\left(F_{p}(x+t c)-F_{p}(x)\right) \\
& =\lim _{t \rightarrow 0} \lim _{n \rightarrow \infty} t^{-1} c^{T}\left(F_{2}\left(x_{n}+t c\right)-F_{2}\left(x_{n}\right)\right) \\
& =\lim _{t \rightarrow 0} \lim _{n \rightarrow \infty} \int_{0}^{1} c^{T} \nabla F_{2}\left(x_{n}+s t c\right) c d s \geq m_{0} c^{T} c \quad \text { for all } c \in \ell^{0} .
\end{aligned}
$$

Thus

$$
c^{T} \nabla F_{p}(x) c \geq m_{0} c^{T} c \quad \text { for all } c \in \ell^{2} \text { and } x \in \ell^{p}
$$

by (4.1), (4.5), the density of $\ell^{0}$ in $\ell^{2}$, and $\mathcal{A}$ is a Banach subalgebra of $\mathcal{B}\left(\ell^{2}\right)$. Hence $\nabla F_{p}(x)$ is invertible on $\ell^{2}$ and $\left\|\left(\nabla F_{p}(x)\right)^{-1}\right\|_{\mathcal{B}\left(\ell^{2}\right)} \leq 1 / m_{0}$ for all $x \in \ell^{p}$. Notice that $\nabla F_{p}(x), x \in \ell^{p}$, is bounded and continuous in $\mathcal{A}$ by (4.5) and the assumption that $\nabla f(x), x \in \ell^{p_{0}}$, is bounded and continuous in $\mathcal{A}$. Then we obtain from the inverseclosedness of the subalgebra $\mathcal{A}$ in $\mathcal{B}\left(\ell^{2}\right)$ that $\left(\nabla F_{p}\right)^{-1}$ is bounded and continuous in $\mathcal{A}$. This together with (2.4) proves that the gradient $\nabla\left(F_{p}^{-1}\right)$ of the inverse function $F_{p}^{-1}$ is bounded in $\mathcal{A}$. The continuity of $\nabla\left(F_{p}^{-1}\right)$ in $\mathcal{A}$ follows from (2.4), the continuity of $\nabla F_{p}$ in $\mathcal{A}$ and the boundedness of $\nabla\left(F_{p}^{-1}\right)$ in $\mathcal{A}$.

(ii) We recall that the function $F_{2}$ satisfies all requirements for the function $f$ in Theorem 3.1. Then by Theorem 3.1, there exists a unique extension $F$ of the function $F_{2}$ on $\ell^{p}, 2 \leq p \leq \infty$, which is continuous with respect to the strong topology of $\ell^{p}$ when $1 \leq p<\infty$ and the weak-star topology of $\ell^{p}$ when $p=\infty$. As shown earlier, $F_{p}, 2 \leq p \leq p_{0}$, are the restriction of the function $f$ on $\ell^{p_{0}}$ and are continuous with respect to the strong topology of $\ell^{p}$ when $1 \leq p<\infty$ and the 
weak-star topology of $\ell^{p}$ when $p=\infty$. Hence $F=F_{p}$ when $2 \leq p \leq p_{0}$, which in turn implies that for $p_{0}<p \leq \infty$, the function $F$ is a continuous extension of the function $f$ on $\ell^{p_{0}}$. The Lipschitz conditions (4.2) and (4.3) for the function $F$ follow from (2.4), Theorem 3.1 and the inclusion $\ell^{2} \subset \ell^{p_{0}}$.

\section{ERror estimate}

In this section, we apply the invertibility theorem (Theorem 4.1) established in the previous section, and discuss the $\ell^{p}$-error estimate for solving the nonlinear functional equation (1.1) in the presence of noises in $\ell^{p}, 1 \leq p \leq \infty$.

Theorem 5.1. Let $2 \leq p_{0} \leq \infty, 1 \leq p \leq p_{0}$, $\mathcal{A}$ be a subalgebra of $\mathcal{B}\left(\ell^{q}\right)$ for all $1 \leq q \leq \infty$ with the property that it admits norm control in $\mathcal{B}\left(\ell^{2}\right)$ and satisfies (3.1), and let $f$ be a continuous function on $\ell^{p_{0}}$ such that $f(0)=0$, its gradient $\nabla f$ is bounded and continuous in $\mathcal{A}$ and satisfies (4.1). If $x_{0}$ and $x_{\epsilon}$ are the solutions of the nonlinear functional equation (1.1) from the noiseless observed data $y \in \ell^{p}$ and the noisy observed data $y+\epsilon$ in $\ell^{p}$ respectively (i.e., $f\left(x_{0}\right)=y$ and $f\left(x_{\epsilon}\right)=y+\epsilon$ ), then

$$
\left\|x_{\epsilon}-x_{0}\right\|_{p} \leq\left(\sup _{z \in \ell^{p_{0}}}\left\|\nabla f^{-1}(z)\right\|_{\mathcal{A}}\right)\left(\sup _{\|A\|_{\mathcal{A}} \leq 1}\|A\|_{\mathcal{B}\left(\ell^{p}\right)}\right)\|\epsilon\|_{p}
$$

and

$$
\frac{\left\|x_{\epsilon}-x_{0}\right\|_{p}}{\left\|x_{0}\right\|_{p}} \leq \kappa_{\mathcal{A}}(f)\left(\sup _{\|A\|_{\mathcal{A}} \leq 1}\|A\|_{\mathcal{B}\left(\ell^{p}\right)}\right)^{2} \frac{\|\epsilon\|_{p}}{\|y\|_{p}}
$$

where $\kappa_{\mathcal{A}}(f)=\left(\sup _{z \in \ell^{p_{0}}}\|\nabla f(z)\|_{\mathcal{A}}\right)\left(\sup _{z \in \ell^{p_{0}}}\left\|\nabla f^{-1}(z)\right\|_{\mathcal{A}}\right)$.

Remark 5.2. If $\mathcal{A}=\mathcal{B}\left(\ell^{2}\right)$ and $f$ is a linear function on $\ell^{p_{0}}$ (i.e, $f(x)=A x$ for some matrix $A)$, then $\kappa_{\mathcal{A}}(f)$ in Theorem 5.1 becomes the condition number for the matrix $A$. So the quantity $\kappa_{\mathcal{A}}(f)$ can be thought as the condition number of the function $f$ in the Banach algebra $\mathcal{A}$.

Remark 5.3. Estimates in Theorems 3.1, 4.1 and 5.1 could be improved by replacing the $\mathcal{A}$-norm by the conventional $\mathcal{B}\left(\ell^{p}\right)$-norm. For instance, we can replace (5.1) and (5.2) in Theorem 5.1 by the following better estimates:

$$
\left\|x_{\epsilon}-x_{0}\right\|_{p} \leq\left(\sup _{z \in \ell^{p} 0}\left\|\nabla f^{-1}(z)\right\|_{\mathcal{B}\left(\ell^{p}\right)}\right)\|\epsilon\|_{p},
$$

and

$$
\frac{\left\|x_{\epsilon}-x_{0}\right\|_{p}}{\left\|x_{0}\right\|_{p}} \leq\left(\sup _{z \in \ell^{p_{0}}}\|\nabla f(z)\|_{\mathcal{B}\left(\ell^{p}\right)}\right)\left(\sup _{z \in \ell^{p_{0}}}\left\|\nabla f^{-1}(z)\right\|_{\mathcal{B}\left(\ell^{p}\right)}\right) \frac{\|\epsilon\|_{p}}{\|y\|_{p}} .
$$

We select the suboptimal estimates in Theorems 3.1, 4.1 and 5.1 for emphasizing the fundamental role the inverse-closed Banach algebra $\mathcal{A}$ has played, and for increasing computational feasibility, c.f. the arguments used to prove Theorems 6.1, 7.1 and 8.1. On the other hand, the replacement of $\mathcal{A}$-norm by the ordinary $\mathcal{B}\left(\ell^{p}\right)$-norm does not always work well. In our study of global linear convergence of the Van-Cittert iteration method (Theorem 6.1), we cannot replace the $\mathcal{A}$-norm in (6.11) by the ordinary $\mathcal{B}\left(\ell^{p}\right)$-norm. The reasons are that the differential norm property (2.2) does 
not hold when $\mathcal{A}$ is replaced by $\mathcal{B}\left(\ell^{p}\right), p \neq 2$, and that the new $b_{j}$ with the $\mathcal{A}$-norm replaced by the $\mathcal{B}\left(\ell^{p}\right)$-norm may not satisfy the crucial iterative properties (6.12) and (6.13).

Remark 5.4. The mean squared error, as a signal fidelity measure, is widely criticized in the field of signal processing for its failure to deal with perceptually important signals such as speech and images. An interesting alternative is the $\ell^{p}$-norm with $p \neq 2$, possibly with adaptive spatial weighting, see the recent review paper [67] on the signal fidelity measure. This is one of our motivations to consider solving the nonlinear functional equation (1.1) in the presence of noises in $\ell^{p}$ with $p \neq 2$.

Proof of Theorem 5.1. By Theorem 4.1, the function $f$ is invertible on $\ell^{p}$ and hence the nonlinear functional equation (1.1) is solvable in $\ell^{p}, 1 \leq p \leq p_{0}$. Thus $x, x_{\epsilon} \in \ell^{p}$.

Again by Theorem 4.1, the inverse of the function $f$ on $\ell^{p}$ has its gradient bounded and continuous in $\mathcal{A}$. Thus

$$
\left\|f^{-1}\left(y_{1}\right)-f^{-1}\left(y_{2}\right)\right\|_{p} \leq\left(\sup _{z \in \ell^{p_{0}}}\left\|\nabla f^{-1}(z)\right\|_{\mathcal{A}}\right)\left(\sup _{\|A\|_{\mathcal{A}} \leq 1}\|A\|_{\mathcal{B}\left(\ell^{p}\right)}\right)\left\|y_{1}-y_{2}\right\|_{p}
$$

for all $y_{1}, y_{2} \in \ell^{p}$. Applying the above estimate with $y_{1}, y_{2}$ replaced by $y, y+\epsilon \in \ell^{p}$ proves the absolute error estimate (5.1).

Using the above argument with the function $f^{-1}$ replaced by the function $f$ leads to

$$
\left\|f\left(x_{1}\right)-f\left(x_{2}\right)\right\|_{p} \leq\left(\sup _{z \in \ell^{p_{0}}}\|\nabla f(z)\|_{\mathcal{A}}\right)\left(\sup _{\|A\|_{\mathcal{A}} \leq 1}\|A\|_{\mathcal{B}\left(\ell^{p}\right)}\right)\left\|x_{1}-x_{2}\right\|_{p}
$$

for all $x_{1}, x_{2} \in \ell^{p}$. Taking $x_{1}=x_{0}$ and $x_{2}=0$ in (5.5) and using $f(0)=0$ gives

$$
\|y\|_{p}=\left\|f\left(x_{0}\right)\right\|_{p} \leq\left(\sup _{z \in \ell^{p} 0}\|\nabla f(z)\|_{\mathcal{A}}\right)\left(\sup _{\|A\|_{\mathcal{A}} \leq 1}\|A\|_{\mathcal{B}\left(\ell^{p}\right)}\right)\left\|x_{0}\right\|_{p} .
$$

Hence the relative error estimate (5.2) follows from (5.1) and (5.6).

\section{Convergence of iteration methods}

In this section, we consider numerically solving the nonlinear functional equation (1.1). We show in Theorem 6.1 that the Van-Cittert iteration method has global exponential convergence in $\ell^{p}$, and in Theorem 6.4 that the quasi-Newton iteration method has local quadratic convergence in $\ell^{p}, 1 \leq p \leq \infty$. The Van-Cittert method is the only iteration method, that we found, which has global exponential convergence in $\ell^{p}, p \neq 2$, for solving infinite-dimensional nonlinear functional equation (1.1). We are seeking to derive other iteration methods to solve the nonlinear functional equation (1.1), that are easily implementable and stable in the presence of noises, and that have global (or local) convergence in $\ell^{p}$ with $p \neq 2$.

\subsection{Global linear convergence of the Van-Cittert iteration method.}

Theorem 6.1. Let $2 \leq p_{0} \leq \infty$, and let $\mathcal{A}$ be a Banach algebra of infinite matrices with the property that it is a subalgebra of $\mathcal{B}\left(\ell^{q}\right)$ for all $1 \leq q \leq \infty$, it contains the identity matrix I, it is closed under the transpose operation, and it satisfies (3.1) and the differential norm inequality (2.2) for some $C_{0} \in(0, \infty)$ and $\theta \in[0,1)$. 
Assume that $f$ is a continuous function on $\ell^{p_{0}}$ such that $f(0)=0$, its gradient $\nabla f$ is bounded and continuous in $\mathcal{A}$ and satisfies the strict monotonicity property (4.1). Set $L=\sup _{z \in \ell^{p_{0}}}\|\nabla f(z)\|_{\mathcal{B}\left(\ell^{2}\right)}$ and let $m_{0}$ be the strict monotonicity constant in (4.1). Given $1 \leq p \leq p_{0}$ and $y \in \ell^{p}$, take an initial guess $x_{0} \in \ell^{p}$ and define $x_{n} \in \ell^{p}, n \geq 1$, iteratively by

$$
x_{n}=x_{n-1}-\alpha\left(f\left(x_{n-1}\right)-y\right), n \geq 1 .
$$

Then for any $0<\alpha<m_{0} /\left(L+L^{2}\right)$, the sequence $x_{n}, n \geq 0$, converges exponentially in $\ell^{p}$ to the unique true solution $f^{-1}(y)$ of the nonlinear functional equation (1.1). Moreover

$$
\left\|x_{n}-f^{-1}(y)\right\|_{p} \leq C_{1}\left\|x_{0}-f^{-1}(y)\right\|_{p} r^{n}, \quad n \geq 0,
$$

where $r>\left(1-\alpha+\alpha^{2} L+\alpha^{2} L^{2}\right) /\left(1-\alpha+\alpha m_{0}\right)$ and $C_{1}$ is a positive constant depending only on $\alpha, r, p$ and $\mathcal{A}$.

Remark 6.2. We may rewrite the Van-Cittert iteration (6.1) as $x_{n}=T x_{n-1}$, where $T x=x-\alpha(f(x)-y), x \in \ell^{p}$. Thus the Van-Cittert iteration (6.1) is a Picard iteration to solve the nonlinear functional equation (1.1). The logic behind the Van-Cittert iteration (6.1) is that we define the new guess by adding the difference $y-f\left(x_{0}\right)$ weighted by a relaxation factor $\alpha$ to the initial guess $x_{0}$ if the difference $y-f\left(x_{0}\right)$ between the observation $f\left(x_{0}\right)$ of the initial guess $x_{0}$ and the observed data $y$ is above a threshold, and we repeat the above iteration until the difference between the observation of the guess and the observed data is below a threshold. The Van-Cittert iteration (6.1) can be easily implemented and is fairly stable in the presence of noises in $\ell^{p}$, see Section 7.3 for numerical simulations.

It has been established in [58, 60] that the Jaffard class $\mathcal{J}_{\beta}(\Lambda)$ in (2.3) satisfies the paracompact condition (2.5). We can mimic the proof there to show that the Jaffard class $\mathcal{J}_{\beta}(\Lambda)$ satisfies the differential norm inequality (2.2). Then we have the following corollary by Theorem 6.1,

Corollary 6.3. Let $\beta>d, \Lambda$ be a relatively-separated subset of $\mathbb{R}^{d}$, and let $p_{0}, p, f, L, \alpha$ be as in Theorem 6.1 with the Banach algebra $\mathcal{A}$ replaced by the Jaffard class $\mathcal{J}_{\beta}(\Lambda)$. Then the sequence $x_{n}, n \geq 0$, in the Van-Cittert iteration (6.1) converges exponentially in $\ell^{p}(\Lambda)$ to the true solution of the nonlinear functional equation (1.1) with the observed data $y$.

Proof of Theorem 6.1. By Proposition 2.4, $\mathcal{A}$ is an inverse-closed Banach subalgebra of $\mathcal{B}\left(\ell^{2}\right)$ that admits norm control. This together with Theorem 4.1 implies that $f$ is invertible on $\ell^{p}$. Hence $x^{*}:=f^{-1}(y) \in \ell^{p}$. For the Van-Cittert iteration (6.1),

$$
\begin{aligned}
x_{n+1}-x^{*}= & (1-\alpha)\left(x_{n}-x^{*}\right)+\alpha\left(x_{n+1}-x^{*}-f\left(x_{n+1}\right)+f\left(x^{*}\right)\right) \\
& -\alpha\left(x_{n+1}-x_{n}-f\left(x_{n+1}\right)+f\left(x_{n}\right)\right) \\
= & (1-\alpha)\left(x_{n}-x^{*}\right)+\alpha\left(x_{n+1}-x^{*}-f\left(x_{n+1}\right)+f\left(x^{*}\right)\right) \\
& +\alpha^{2}\left(f\left(x_{n}\right)-f\left(x^{*}\right)\right)+\alpha\left(f\left(x_{n}-\alpha\left(f\left(x_{n}\right)-f\left(x^{*}\right)\right)\right)-f\left(x_{n}\right)\right) .
\end{aligned}
$$


This leads to the following crucial equation:

$$
A_{n}\left(x_{n+1}-x^{*}\right)=B_{n}\left(x_{n}-x^{*}\right) \text { for all } n \geq 0,
$$

where

$$
A_{n}=(1-\alpha) I+\alpha \int_{0}^{1} \nabla f\left(t x_{n+1}+(1-t) x^{*}\right) d t
$$

and

$$
\begin{aligned}
B_{n}= & (1-\alpha) I+\alpha^{2}\left(I-\int_{0}^{1} \nabla f\left(x_{n}-s \alpha\left(f\left(x_{n}\right)-f\left(x^{*}\right)\right)\right) d s\right) \\
& \times\left(\int_{0}^{1} \nabla f\left(t x_{n}+(1-t) x^{*}\right) d t\right), \quad n \geq 0 .
\end{aligned}
$$

For infinite matrices $A_{n}$ and $B_{n}, n \geq 0$, we obtain from (4.1), (6.4) and (6.5) that

$$
\left\{\begin{array}{l}
\left\|A_{n}\right\|_{\mathcal{B}\left(\ell^{2}\right)} \leq 1+(L-1) \alpha, \\
\left\|A_{n}\right\|_{\mathcal{A}} \leq(1-\alpha)\|I\|_{\mathcal{A}}+\alpha \sup _{z \in \ell^{p_{0}}}\|\nabla f(z)\|_{\mathcal{A}}, \\
c^{T} A_{n} c \geq\left(1-\alpha+\alpha m_{0}\right) c^{T} c \text { for all } c \in \ell^{2},
\end{array}\right.
$$

and

$$
\left\{\begin{aligned}
\left\|B_{n}\right\|_{\mathcal{B}\left(\ell^{2}\right)} \leq & 1-\alpha+\alpha^{2} L(1+L) \\
\left\|B_{n}\right\|_{\mathcal{A}} \leq(1-\alpha)\|I\|_{\mathcal{A}}+\alpha^{2}\left(\sup _{z \in \ell^{p_{0}}}\|\nabla f(z)\|_{\mathcal{A}}\right) & \\
& \times\left(\|I\|_{\mathcal{A}}+\sup _{z \in \ell^{p_{0}}}\|\nabla f(z)\|_{\mathcal{A}}\right) .
\end{aligned}\right.
$$

For every $n \geq 0$, it follows from (6.6) that $A_{n}$ is invertible in $\ell^{2}$ and the operator norm $\left\|\left(A_{n}\right)^{-1}\right\|_{\mathcal{B}\left(\ell^{2}\right)}$ of its inverse $\left(A_{n}\right)^{-1}$ is bounded by $\left(1-\alpha+\alpha m_{0}\right)^{-1}$. The above property for infinite matrices $A_{n}, n \geq 0$, together with (6.7) and the norm control property of the Banach algebra $\mathcal{A}$ given in Proposition 2.4, implies that

$$
\left\|A_{n}^{-1} B_{n}\right\|_{\mathcal{B}\left(\ell^{2}\right)}=\frac{1-\alpha+\alpha^{2} L(1+L)}{1-\alpha+\alpha m_{0}}:=r_{1}<1
$$

and

$$
\left\|A_{n}^{-1} B_{n}\right\|_{\mathcal{A}} \leq D_{1}<\infty \quad \text { for all } n \geq 0
$$

where $D_{1}$ is a positive constant independent of $n \geq 0$.

Now we rewrite $(6.3)$ as

$$
x_{n+1}-x^{*}=A_{n}^{-1} B_{n}\left(x_{n}-x^{*}\right) .
$$

Applying the above formula iteratively gives

$$
x_{n+1}-x^{*}=A_{n}^{-1} B_{n} A_{n-1}^{-1} B_{n-1} \cdots A_{0}^{-1} B_{0}\left(x_{0}-x^{*}\right) .
$$

Then the proof of the exponential convergence of $x_{n}, n \geq 0$, to $x^{*}$ reduces to showing that there exist positive constant $C \in(0,+\infty)$ and $r \in(0,1)$ such that

$$
\left\|A_{n}^{-1} B_{n} A_{n-1}^{-1} B_{n-1} \cdots A_{0}^{-1} B_{0}\right\|_{\mathcal{A}} \leq C r^{n}, \quad n \geq 0 .
$$

For $\theta \in(0,1)$, set

$$
b_{j}=\left(2 C_{0}\right)^{1 / \theta} r_{1}^{-j} \sup _{k \geq 0}\left\|A_{j+k-1}^{-1} B_{j+k-1} \cdots A_{k}^{-1} B_{k}\right\|_{\mathcal{A}},
$$


where $C_{0}$ and $r_{1}$ are the constants in (2.2) and (6.8) respectively. Then we obtain from (6.8), (6.9) and the differential norm property (2.2) that

$$
\begin{aligned}
b_{2 j}= & \left(2 C_{0}\right)^{1 / \theta} r_{1}^{-2 j} \sup _{k \geq 0}\left\|A_{2 j+k-1}^{-1} B_{2 j+k-1} \cdots A_{k}^{-1} B_{k}\right\|_{\mathcal{A}} \\
\leq & \left(2 C_{0}\right)^{(1+\theta) / \theta} r_{1}^{-2 j} \max \left(\left\|A_{2 j+k-1}^{-1} B_{2 j+k-1} \cdots A_{j+k}^{-1} B_{j+k}\right\|_{\mathcal{A}}^{1+\theta}\right. \\
& \left.\quad\left\|A_{j+k-1}^{-1} B_{j+k-1} \cdots A_{k}^{-1} B_{k}\right\|_{\mathcal{A}}^{1+\theta}\right) \\
& \times \max \left(\left\|A_{2 j+k-1}^{-1} B_{2 j+k-1} \cdots A_{j+k}^{-1} B_{j+k}\right\|_{\mathcal{B}\left(\ell^{2}\right)}^{1-\theta},\right. \\
\leq & \left.\quad\left\|A_{j+k-1}^{-1} B_{j+k-1} \cdots A_{k}^{-1} B_{k}\right\|_{\mathcal{B}\left(\ell^{2}\right)}^{1-\theta}\right)
\end{aligned}
$$

and

$$
\begin{aligned}
b_{2 j+1} & \leq\left(2 C_{0}\right)^{1 / \theta} r_{1}^{-2 j-1} \sup _{k \geq 0}\left\|A_{2 j+k}^{-1} B_{2 j+k}\right\|_{\mathcal{A}}\left\|A_{2 j+k-1}^{-1} B_{2 j+k-1} \cdots A_{k}^{-1} B_{k}\right\|_{\mathcal{A}} \\
& \leq\left(D_{1} / r_{1}\right) b_{2 j} \leq\left(D_{1} / r_{1}\right) b_{j}^{1+\theta}
\end{aligned}
$$

for all $j \geq 1$. Applying (6.12) and (6.13) repeatedly yields

$$
\begin{aligned}
b_{n} & \leq\left(D_{1} / r\right)^{\epsilon_{0}} b_{\sum_{i=1}^{l} \epsilon_{i} 2^{2-1}}^{1+\theta} \leq \cdots \leq\left(D_{1} / r\right)^{\sum_{i=0}^{l-1} \epsilon_{i}(1+\theta)^{i}} b_{\epsilon_{l}}^{(1+\theta)^{l}} \\
& \leq\left(D_{1} / r\right)^{(1+\theta)^{l} / \theta}\left(\left(2 C_{0}\right)^{1 / \theta} D_{1} / r\right)^{(1+\theta)^{l}} \leq\left(2 C_{0} D_{1} / r_{1}\right)^{(1+\theta) n^{\log _{2}(1+\theta) / \theta}},
\end{aligned}
$$

where $n=\sum_{i=0}^{l} \epsilon_{i} 2^{i}$ with $\epsilon_{i} \in\{0,1\}$ and $\epsilon_{l}=1$. Therefore for $\theta \in(0,1)$,

$$
\begin{aligned}
\left\|A_{n}^{-1} B_{n} A_{n-1}^{-1} B_{n-1} \cdots A_{0}^{-1} B_{0}\right\|_{\mathcal{A}} & \leq\left(2 C_{0}\right)^{-1 / \theta} r_{1}^{n} b_{n} \\
& \leq\left(2 C_{0}\right)^{-1 / \theta} r_{1}^{n}\left(C_{0} D_{1} / r_{1}\right)^{(1+\theta) n^{\log _{2}(1+\theta)} / \theta}
\end{aligned}
$$

for all $n \geq 1$.

For $\theta=0$, we can mimic the above argument to obtain that

$$
\left\|A_{n}^{-1} B_{n} A_{n-1}^{-1} B_{n-1} \cdots A_{0}^{-1} B_{0}\right\|_{\mathcal{A}} \leq r_{1}^{n}\left(2 C_{0} D_{1} / r_{1}\right)^{1+\log _{2} n} \quad \text { for all } n \geq 1 .
$$

Hence (6.10) follows from (6.14) and (6.15) by letting $r \in\left(r_{1}, 1\right)$, and the exponential convergence of $x_{n}$ in $\ell^{p}$ is established.

6.2. Local quadratic convergence of the quasi-Newton method. In this subsection, we show that the quasi-Newton iterative method (6.17) has local Rquadratic convergence in $\ell^{p}$.

Theorem 6.4. Let $2 \leq p_{0} \leq \infty$, let $\mathcal{A}$ be a subalgebra of $\mathcal{B}\left(\ell^{q}\right)$ for all $1 \leq q \leq \infty$ and an subalgebra of $\mathcal{B}\left(\ell^{2}\right)$ that admits norm control, and satisfy (3.1), and let $f$ be a continuous function on $\ell^{p_{0}}$ such that $f(0)=0$, its gradient $\nabla f$ is bounded and continuous in $\mathcal{A}$, and satisfies the strictly monotonic property (4.1) and

$$
\left\|\nabla f(x)-\nabla f\left(x^{\prime}\right)\right\|_{\mathcal{A}} \leq C_{1}\left\|x-x^{\prime}\right\|_{p_{0}} \quad \text { for all } x, x^{\prime} \in \ell^{p_{0}}
$$

where $C_{1} \in(0, \infty)$. Given an observed data $y \in \ell^{p}$ and an initial guess $x_{0} \in \ell^{p}, 1 \leq$ $p \leq p_{0}$, define the quasi-Newton iteration by

$$
x_{n+1}=x_{n}-\left(\nabla f\left(x_{n}\right)\right)^{-1}\left(f\left(x_{n}\right)-y\right), \quad n \geq 1 .
$$


Then $x_{n}, n \geq 1$, converges $R$-quadratically to the solution $f^{-1}(y)$ of the nonlinear functional equation (1.1) with the observed data $y$ as there exists a positive constant $C$ such that

$$
\left\|x_{n+1}-x_{n}\right\|_{p} \leq C\left\|x_{n}-x_{n-1}\right\|_{p}^{2} \text { for all } n \geq 1,
$$

provided that the initial guess $x_{0}$ is sufficiently close to the true solution $f^{-1}(y)$.

By Theorems 4.1 and 6.4, we have the following corollary.

Corollary 6.5. Let $\beta>d, \Lambda$ be a relatively-separated subset of $\mathbb{R}^{d}, \mathcal{A}$ be the Jaffard class $\mathcal{J}_{\beta}(\Lambda)$, and let $p_{0}$ and $f$ be as in Theorem 6.4. Then the quasi-Newton iteration (6.17) has local $R$-quadratical convergence in $\ell^{p}, 1 \leq p \leq p_{0}$.

Proof of Theorem 6.4. By (6.16) and (6.17),

$$
\left\|x_{1}-x_{0}\right\|_{p} \leq\left(\sup _{z \in \ell^{p_{0}}}\left\|(\nabla f(z))^{-1}\right\|_{\mathcal{A}}\right)\left(\sup _{\|A\|_{\mathcal{A}} \leq 1}\|A\|_{\mathcal{B}\left(\ell^{p}\right)}\right)\left\|f\left(x_{0}\right)-y\right\|_{p}
$$

and

$$
\begin{aligned}
\left\|x_{n+1}-x_{n}\right\|_{p}= & \left\|\left(\nabla f\left(x_{n}\right)\right)^{-1}\left(f\left(x_{n}\right)-y\right)\right\|_{p} \\
\leq & \left(\sup _{z \in \ell^{p} 0}\left\|(\nabla f(z))^{-1}\right\|_{\mathcal{A}}\right)\left(\sup _{\|A\|_{\mathcal{A}} \leq 1}\|A\|_{\mathcal{B}\left(\ell^{p}\right)}\right) \\
& \times\left\|f\left(x_{n}\right)-f\left(x_{n-1}\right)-\nabla f\left(x_{n-1}\right)\left(x_{n}-x_{n-1}\right)\right\|_{p} \\
\leq & C_{1}\left(\sup _{z \in \ell^{p}}\left\|(\nabla f(z))^{-1}\right\|_{\mathcal{A}}\right)\left(\sup _{\|A\|_{\mathcal{A}} \leq 1}\|A\|_{\mathcal{B}\left(\ell^{p}\right)}\right)\left\|x_{n}-x_{n-1}\right\|_{p}^{2}
\end{aligned}
$$

for all $n \geq 1$. This proves the local R-quadratic convergence of the quasi-Newton iteration method.

\section{INSTANTANEOUS COMPANDING AND AVERAGE SAMPLING}

In this section, the theory established in previous sections for the nonlinear functional equation (1.1) will be used to handle the nonlinear sampling procedure (1.7) of instantaneous companding and subsequently average sampling. In the first subsection, we apply the invertibility theorem (Theorem 4.1) to establish $\ell^{p}$-stability of the nonlinear sampling procedure (1.7), while in the second subsection we use the Van-Cittert iteration method in Theorem 6.1 and the quasi-Newton iteration method in Theorem 6.4 to recover signals from averaging samples of their instantaneous companding. We present some numerical simulations in the last subsection to demonstrate the stable signal recovery in the presence of bounded noises.

7.1. $\ell^{p}$-stability of the nonlinear sampling procedure (1.7). We say that $\Phi=$ $\left(\phi_{\lambda}\right)_{\lambda \in \Lambda}$ is a Riesz basis for the space $V_{p}(\Phi)$ in (1.9) if

$$
0<\inf _{\|c\|_{p}=1}\left\|c^{T} \Phi\right\|_{p} \leq \sup _{\|c\|_{p}=1}\left\|c^{T} \Phi\right\|_{p}<\infty .
$$

Given two closed subspaces $V$ and $W$ of $L^{2}$, define the $g a p \delta(V, W)$ from $V$ to $W$ by

$$
\delta(V, W)=\sup _{\|h\|_{2} \leq 1, h \in V} \inf _{\tilde{h} \in W}\|h-\tilde{h}\|_{2}=\sup _{\|h\|_{2} \leq 1, h \in V}\left\|h-P_{W} h\right\|_{2},
$$


where $P_{W}$ is the projection operator onto $W$.

Theorem 7.1. Let $\Lambda, \Gamma$ be two relatively-separated subsets of $\mathbb{R}^{d}$, and let $\Phi:=$ $\left(\phi_{\lambda}\right)_{\lambda \in \Lambda}$ and $\Psi:=\left(\psi_{\gamma}\right)_{\gamma \in \Gamma}$ have polynomial decay of order $\beta>d$, i.e.,

$$
\sup _{\lambda \in \Lambda, x \in \mathbb{R}^{d}}|\phi(x)|(1+|x-\lambda|)^{\beta}+\sup _{\gamma \in \Gamma, x \in \mathbb{R}^{d}}\left|\psi_{\gamma}(x)\right|(1+|x-\gamma|)^{\beta}<\infty,
$$

and generate Riesz bases for $V_{2}(\Phi)$ and $V_{2}(\Psi)$ respectively. If the gap $\delta\left(V_{2}(\Phi), V_{2}(\Psi)\right)$ from $V_{2}(\Phi)$ to $V_{2}(\Psi)$ is strictly less than one, and the companding function $F$ is a continuously differential function on $\mathbb{R}$ satisfying $F(0)=0$ and

$$
\mu:=\sup _{t \in \mathbb{R}}\left|1-m F^{\prime}(t)\right|<\frac{\sqrt{1-\left(\delta\left(V_{2}(\Phi), V_{2}(\Psi)\right)\right)^{2}}}{\delta\left(V_{2}(\Phi), V_{2}(\Psi)\right)+\sqrt{1-\left(\delta\left(V_{2}(\Phi), V_{2}(\Psi)\right)\right)^{2}}}
$$

for some nonzero constant $m$, then the nonlinear sampling procedure $S_{F, \Psi}$ in (1.7) is stable on $V_{p}(\Phi), 1 \leq p \leq \infty$, i.e., there exist positive constants $C_{1}$ and $C_{2}$ such that

$$
C_{1}\left\|h_{1}-h_{2}\right\|_{p} \leq\left\|S_{F, \Psi}\left(h_{1}\right)-S_{F, \Psi}\left(h_{2}\right)\right\|_{p} \leq C_{2}\left\|h_{1}-h_{2}\right\|_{p}
$$

for all $h_{1}, h_{2} \in V_{p}(\Phi)$.

Remark 7.2. We remark that, in Theorem 7.1, the assumption that the gap $\delta\left(V_{2}(\Phi), V_{2}(\Psi)\right)$ from $V_{2}(\Phi)$ to $V_{2}(\Psi)$ is strictly less than one is a necessary and sufficient condition for the $\ell^{2}$-stability of the sampling procedure (1.7) without instantaneous companding (i.e., $F(t) \equiv t$ ) on $V_{2}(\Phi)$. The above equivalence follows from (7.6), (7.8) and (7.12). For the sampling procedure (1.7) without instantaneous companding, the readers may refer to [7, 59] and references therein.

As a stable nonlinear sampling procedure is one-to-one, from Theorem 7.1 we obtain the uniqueness of the nonlinear sampling procedure (1.7), which is established in [19] for $p=2$ when $\mu<\frac{1-\delta\left(V_{2}(\Phi), V_{2}(\Psi)\right)}{1+\delta\left(V_{2}(\Phi), V_{2}(\Psi)\right)}$, a stronger assumption on $F$ than (7.3) in Theorem 7.1 .

Corollary 7.3. Let $\Phi, \Psi$ and $F$ be as in Theorem 7.1. Then any signal $h \in$ $V_{p}(\Phi), 1 \leq p \leq \infty$, is uniquely determined by its nonlinear sampling data $\langle F(h), \Psi\rangle$.

Notice that $\delta(V, V)=0$ for any closed subspace $V$ of $L^{2}$. Then applying Theorem 7.1 with the generator $\Phi$ of the space $V_{2}(\Phi)$ being taken as the average sampler $\Psi$ in the nonlinear sampling procedure (1.7), we obtain the following result.

Corollary 7.4. Let $\Lambda$ be a relatively-separated subset of $\mathbb{R}^{d}$, and let $\Phi:=\left(\phi_{\lambda}\right)_{\lambda \in \Lambda}$ have polynomial decay of order $\beta>d$ and generate a Riesz basis for $V_{2}(\Phi)$. If the companding function $F$ has its derivative bounded away from zero and infinity, then the nonlinear sampling procedure $S_{F, \Psi}$ in (1.7) with $\Psi=\Phi$ is stable on $V_{p}(\Phi), 1 \leq$ $p \leq \infty$. 
Remark 7.5. Notice that

$$
\begin{aligned}
\left(\inf _{s \in \mathbb{R}} F^{\prime}(s)\right)\left\|c_{1}-c_{2}\right\|_{2}^{2} & \leq\left(c_{1}-c_{2}\right)^{T}\left(S_{F, \Phi}\left(c_{1}^{T} \Phi\right)-S_{F, \Phi}\left(c_{2}^{T} \Phi\right)\right) \\
& =\int_{\mathbb{R}^{d}}\left(F\left(c_{1}^{T} \Phi(t)\right)-F\left(c_{2}^{T} \Phi(t)\right)\right)\left(c_{1}^{T} \Phi(t)-c_{2}^{T} \Phi(t)\right) d t \\
& \leq\left(\sup _{s \in \mathbb{R}} F^{\prime}(s)\right)\left\|c_{1}-c_{2}\right\|_{2}^{2}
\end{aligned}
$$

for all $c_{1}, c_{2} \in V_{2}(\Phi)$. Hence the stability conclusion in Corollary 7.4 holds for $p=2$ without the polynomial decay assumption on $\Phi$. This is established for signals living in the Paley-Wiener space [40, 53] or a shift-invariant space [19].

Given relatively-separated subsets $\Lambda$ and $\Gamma$, let the Jaffard class $\mathcal{J}_{\beta}(\Gamma, \Lambda)$ be the family of infinite matrices $A:=(a(\gamma, \lambda))_{\gamma \in \Gamma, \lambda \in \Lambda}$ such that

$$
\|A\|_{\mathcal{J}_{\beta}(\Gamma, \Lambda)}:=\sup _{\gamma \in \Gamma, \lambda}(1+|\gamma-\lambda|)^{\beta}|a(\gamma, \lambda)|<\infty .
$$

Given two vectors $\Phi:=\left(\phi_{\lambda}\right)_{\lambda \in \Lambda}$ and $\Psi:=\left(\psi_{\gamma}\right)_{\gamma \in \Gamma}$ of square-integrable functions, we define their inter-correlation matrix $A_{\Phi, \Psi}$ by $A_{\Phi, \Psi}=\left(\left\langle\phi_{\lambda}, \psi_{\gamma}\right\rangle\right)_{\lambda \in \Lambda, \gamma \in \Gamma}$. To prove Theorem 7.1, we need a technical lemma.

Lemma 7.6. Let $\Lambda, \Gamma$ be relatively-separated subsets of $\mathbb{R}^{d}, \Phi:=\left(\phi_{\lambda}\right)_{\lambda \in \Lambda}$ and $\Psi:=$ $\left(\psi_{\gamma}\right)_{\gamma \in \Gamma}$ have polynomial decay of order $\beta>d$ and generate Riesz bases of $V_{2}(\Phi)$ and $V_{2}(\Psi)$ respectively, and let the function $F$ satisfy $F(0)=0$ and have its derivative being continuous and bounded. Then

(i) $\Phi$ and $\Psi$ generate Riesz bases for $V_{p}(\Phi)$ and $V_{p}(\Psi)$ respectively, where $1 \leq$ $p \leq \infty$.

(ii) The function $f_{F, \Phi, \Psi}$ in (1.10) is well-defined on $\ell^{p}$ for all $1 \leq p \leq \infty$, and has its gradient $\nabla f_{F, \Phi, \Psi}$ being continuous and bounded in the Jaffard class $\mathcal{J}_{\beta}(\Gamma, \Lambda)$.

Proof. (i) We follow the arguments in [61]. By the polynomial decay property for $\Phi$ and relatively-separatedness of the index set $\Lambda$, we have that

$$
\sup _{\|c\|_{p}=1}\left\|c^{T} \Phi\right\|_{p}<\infty .
$$

By Riesz basis property for $V_{2}(\Phi)$ and the polynomial decay property for $\Phi$,

$$
\left(A_{\Phi, \Phi}\right)^{-1} \in \mathcal{B}\left(\ell^{2}(\Lambda)\right) \text { and } A_{\Phi, \Phi} \in \mathcal{J}_{\beta}(\Lambda) .
$$

Hence $\left(A_{\Phi, \Phi}\right)^{-1}$ belong to the same Jaffard class $\mathcal{J}_{\beta}(\Lambda)$ by the inverse-closedness of the Jaffard class $\mathcal{J}_{\beta}(\Lambda)$ in $\mathcal{B}\left(\ell^{2}(\Lambda)\right.$ ), which together with the polynomial decay property of $\Phi$ implies that the dual Riesz basis $\left(A_{\Phi, \Phi}\right)^{-1} \Phi$ has the polynomial decay of same order $\beta$. Hence $c=\left\langle c^{T} \Phi,\left(A_{\Phi, \Phi}\right)^{-1} \Phi\right\rangle$ for all $c \in \ell^{p}$, which yields

$$
\inf _{\|c\|_{p}=1}\left\|c^{T} \Phi\right\|_{p}>0
$$

Combining (7.5) and (7.7) proves the Riesz property for $V_{p}(\Phi)$. 
The Riesz property for $V_{p}(\Psi)$ can be proved by using the same argument to establish (7.5) and (7.7) except with $\Phi$ replaced by $\Psi, \Lambda$ by $\Gamma,(7.6)$ by

$$
\left(A_{\Psi, \Psi}\right)^{-1} \in \mathcal{B}\left(\ell^{2}(\Gamma)\right) \text { and } A_{\Psi, \Psi} \in \mathcal{J}_{\beta}(\Gamma),
$$

which follows from Riesz basis property for $V_{2}(\Psi)$ and the polynomial decay property for $\Psi$.

(ii) For $c=(c(\lambda))_{\lambda \in \Lambda} \in \ell^{p}(\Lambda)$, write $f_{F, \Phi, \Psi}(c)=(d(\gamma))_{\gamma \in \Gamma}$. Then

$$
\begin{aligned}
|d(\gamma)| & \leq \int_{\mathbb{R}^{d}}\left|F\left(c^{T} \Phi(t)\right) \psi_{\gamma}(t)\right| d t \\
& \leq\left\|F^{\prime}\right\|_{\infty}\|\Phi\|_{\infty, \beta}\|\Psi\|_{\infty, \beta} \sum_{\lambda \in \Lambda}|c(\lambda)| \int_{\mathbb{R}^{d}}(1+|t-\lambda|)^{-\beta}(1+|t-\gamma|)^{-\beta} d t \\
& \leq C\left\|F^{\prime}\right\|_{\infty}\|\Phi\|_{\infty, \beta}\|\Psi\|_{\infty, \beta} \sum_{\lambda \in \Lambda}|c(\lambda)|(1+|\lambda-\gamma|)^{-\beta} \quad \text { for all } \gamma \in \Gamma,
\end{aligned}
$$

where $C$ is a positive constant. Thus $f_{F, \Phi, \Psi}(c) \in \ell^{p}(\Gamma)$ for all $c \in \ell^{p}(\Lambda)$, which proves that the function $f_{F, \Phi, \Psi}$ is well-defined on $\ell^{p}(\Lambda)$.

By direct calculation, we have that

$$
\nabla f_{F, \Phi, \Psi}(c)=\left(\left\langle F^{\prime}\left(c^{T} \Phi\right) \phi_{\lambda}, \psi_{\gamma}\right\rangle\right)_{\gamma \in \Gamma, \lambda \in \Lambda} \quad \text { for all } c \in \ell^{p},
$$

and $\lim _{\tilde{c} \rightarrow c \text { in } \ell^{p}}\left\|\tilde{c}^{T} \Phi-c^{T} \Phi\right\|_{\infty}=0$. Thus $\nabla f_{F, \Phi, \Psi}(c), c \in \ell^{p}$, is bounded and continuous in $\mathcal{J}_{\beta}(\Gamma, \Lambda)$.

Proof of Theorem 7.1. By the polynomial decay property for $\Phi$ and $\Psi, A_{\Phi, \Phi} \in$ $\mathcal{J}_{\beta}(\Lambda, \Lambda), A_{\Psi, \Psi} \in \mathcal{J}_{\beta}(\Gamma, \Gamma), A_{\Psi, \Phi} \in \mathcal{J}_{\beta}(\Gamma, \Lambda)$ and $A_{\Phi, \Psi} \in \mathcal{J}_{\beta}(\Lambda, \Gamma)$. From the argument in Lemma 7.6, we have that $\left(A_{\Phi, \Phi}\right)^{-1} \in \mathcal{J}_{\beta}(\Lambda, \Lambda)$ and $\left(A_{\Psi, \Psi}\right)^{-1} \in \mathcal{J}_{\beta}(\Gamma, \Gamma)$. Thus

$$
A_{\Phi, \Psi}\left(A_{\Psi, \Psi}\right)^{-1} A_{\Psi, \Phi} \in \mathcal{J}_{\beta}(\Lambda, \Lambda) .
$$

By the definition of the gap $\delta\left(V_{2}(\Phi), V_{2}(\Psi)\right)$ from $V_{2}(\Phi)$ to $V_{2}(\Psi)$,

$$
\left(1-\left(\delta\left(V_{2}(\Phi), V_{2}(\Psi)\right)\right)^{2}\right) c^{T} A_{\Phi, \Phi} c \leq c^{T} A_{\Phi, \Psi}\left(A_{\Psi, \Psi}\right)^{-1} A_{\Psi, \Phi} c \leq c^{T} A_{\Phi, \Phi} c
$$

for all $c \in \ell^{2}(\Lambda)$. Combining (7.6), (7.10) and (7.11), and applying Wiener's lemma for infinite matrices in the Jaffard class $\mathcal{J}_{\beta}(\Lambda, \Lambda)$ [32, 58, 60], we obtain that $\left(A_{\Phi, \Psi}\left(A_{\Psi, \Psi}\right)^{-1} A_{\Psi, \Phi}\right)^{-1} \in \mathcal{J}_{\beta}(\Lambda, \Lambda)$. Thus the reconstruction matrix

$$
R_{\Phi, \Psi}:=A_{\Phi, \Phi}\left(A_{\Phi, \Psi}\left(A_{\Psi, \Psi}\right)^{-1} A_{\Psi, \Phi}\right)^{-1} A_{\Phi, \Psi}\left(A_{\Psi, \Psi}\right)^{-1} \in \mathcal{J}_{\beta}(\Lambda, \Gamma) .
$$

Define a function $g_{F, \Phi, \Psi}$ on $\ell^{p}(\Lambda)$ by

$$
g_{F, \Phi, \Psi}(x):=R_{\Phi, \Psi} f_{F, \Phi, \Psi}(x), x \in \ell^{p},
$$

where $f_{F, \Phi, \Psi}$ is given in (1.10). Then the function $g_{F, \Phi, \Psi}$ is well-defined on $\ell^{p}(\Lambda)$ and satisfies $g(0)=0$, and its gradient

$$
\nabla g_{F, \Phi, \Psi}(x)=R_{\Phi, \Psi}\left\langle\Psi, F^{\prime}\left(x^{T} \Phi\right) \Phi\right\rangle, x \in \ell^{p},
$$


is continuous and bounded in the Jaffard class $\mathcal{J}_{\beta}(\Lambda, \Lambda)$ by Lemma 7.6. From (7.11) it follow that

$$
\begin{aligned}
c^{T} A_{\Phi, \Phi} c & \leq c^{T} A_{\Phi, \Phi}\left(A_{\Phi, \Psi}\left(A_{\Psi, \Psi}\right)^{-1} A_{\Psi, \Phi}\right)^{-1} A_{\Phi, \Phi} c \\
& =c^{T} R_{\Phi, \Psi} A_{\Psi, \Psi}\left(R_{\Phi, \Psi}\right)^{T} c=\left\|c^{T} R_{\Phi, \Psi} \Psi\right\|_{2}^{2} \\
& \leq\left(1-\left(\delta\left(V_{2}(\Phi), V_{2}(\Psi)\right)\right)^{2}\right)^{-1} c^{T} A_{\Phi, \Phi} c \quad \text { for all } c \in \ell^{2}(\Lambda) .
\end{aligned}
$$

This together with (17.3) implies that

$$
\begin{aligned}
d^{T} \nabla g_{F, \Phi, \Psi}(x) c & =\left\langle F^{\prime}\left(x^{T} \Phi\right)\left(c^{T} \Phi\right), d^{T} R_{\Phi, \Psi} \Psi\right\rangle \\
& \leq m^{-1}(1+\mu)\left\|c^{T} \Phi\right\|_{2}\left\|d^{T} R_{\Phi, \Psi} \Psi\right\|_{2} \\
& \leq m^{-1} B_{1}(1+\mu)\left(1-\left(\delta\left(V_{2}(\Phi), V_{2}(\Psi)\right)\right)^{2}\right)^{-1 / 2}\|c\|_{2}\|d\|_{2}
\end{aligned}
$$

for any $x \in \ell^{p}(\Lambda)$ and $c, d \in \ell^{2}(\Lambda)$, where $B_{1}=\sup _{\|c\|_{2}=1}\left\|c^{T} \Phi\right\|_{2}$. This proves that

$$
\left\|\nabla g_{F, \Phi, \Psi}(x)\right\|_{\mathcal{B}\left(\ell^{2}(\Lambda)\right)} \leq m^{-1} B_{1} \frac{1+\mu}{\sqrt{1-\left(\delta\left(V_{2}(\Phi), V_{2}(\Psi)\right)^{2}\right.}} \quad \text { for all } x \in \ell^{p}(\Lambda)
$$

Notice that

$$
P_{V_{2}(\Phi)}\left(c^{T} R_{\Phi, \Psi} \Psi\right)=c^{T} R_{\Phi, \Psi} A_{\Psi, \Phi} A_{\Phi, \Phi}^{-1} \Phi=c^{T} \Phi
$$

for all $c \in \ell^{2}$ by direct calculation. Hence for any $x \in \ell^{p}$ and $c \in \ell^{2}$,

$$
\begin{aligned}
c^{T} \nabla g_{F, \Phi, \Psi}(x) c & =\left\langle F^{\prime}\left(x^{T} \Phi\right)\left(c^{T} \Phi\right), c^{T} \Phi\right\rangle+\left\langle F^{\prime}\left(x^{T} \Phi\right)\left(c^{T} \Phi\right), c^{T} R_{\Phi, \Psi} \Psi-c^{T} \Phi\right\rangle \\
& \geq m^{-1}(1-\mu)\left\|c^{T} \Phi\right\|_{2}^{2}-m^{-1} \mu\left\|c^{T} \Phi\right\|_{2}\left\|c^{T} R_{\Phi, \Psi} \Psi-c^{T} \Phi\right\|_{2} \\
& \geq m^{-1}(1-\mu-\mu \tan \theta) c^{T} A_{\Phi, \Phi} c \\
& \geq m^{-1} A_{1}(1-\mu-\mu \tan \theta) c^{T} c
\end{aligned}
$$

by (17.3) and (7.13), where $A_{1}=\inf _{\|c\|_{2}=1}\left\|c^{T} \Phi\right\|_{2}$ and

$$
\tan \theta=\delta\left(V_{2}(\Phi), V_{2}(\Psi)\right) / \sqrt{1-\left(\delta\left(V_{2}(\Phi), V_{2}(\Psi)\right)\right)^{2}} .
$$

By the above argument, we see that the function $g_{F, \Phi, \Psi}$ satisfies all requirements for the function $f$ in Theorem 4.1. Thus the function $g_{F, \Phi, \Psi}$ is invertible in $\ell^{p}$ and the gradient of its inverse is bounded and continuous in $\mathcal{J}_{\beta}(\Lambda)$. Then there exists a positive constant $C_{0}$ such that

$$
\left\|c_{1}-c_{2}\right\|_{p} \leq C_{0}\left\|g_{F, \Phi, \Psi}\left(c_{1}\right)-g_{F, \Phi, \Psi}\left(c_{2}\right)\right\|_{p} \text { for all } c_{1}, c_{2} \in \ell^{p} .
$$

Hence for any $h_{1}=c_{1}^{T} \Phi$ and $h_{2}=c_{2}^{T} \Phi \in V_{p}(\Phi)$,

$$
\begin{aligned}
\left\|h_{1}-h_{2}\right\|_{p} & \leq\left(\sup _{\|c\|_{p}=1}\left\|c^{T} \Phi\right\|_{p}\right)\left\|c_{1}-c_{2}\right\|_{p} \leq C\left\|R_{\Phi, \Psi}\left(S_{F, \Psi}\left(h_{1}\right)-S_{F, \Psi}\left(h_{2}\right)\right)\right\|_{p} \\
& \leq C\left\|S_{F, \Psi}\left(h_{1}\right)-S_{F, \Psi}\left(h_{2}\right)\right\|_{p}
\end{aligned}
$$

by (7.12), (17.18) and Lemma 7.6. This proves the first inequality in (7.4).

The second inequality in (7.4) follows from (7.12) and Lemma 7.6. 


\subsection{Reconstructing signals from averaging samples of their instantaneous companding.}

Theorem 7.7. Let $\Lambda, \Gamma$ be two relatively-separated subsets of $\mathbb{R}^{d}, \Phi:=\left(\phi_{\lambda}\right)_{\lambda \in \Lambda}$ and $\Psi:=\left(\psi_{\gamma}\right)_{\gamma \in \Gamma}$ have polynomial decay of order $\beta>d$ and generate Riesz bases for $V_{2}(\Phi)$ and $V_{2}(\Psi)$ respectively, the gap $\delta\left(V_{2}(\Phi), V_{2}(\Psi)\right)$ from $V_{2}(\Phi)$ to $V_{2}(\Psi)$ be strictly less than one, the companding function $F$ be a continuously differential function on $\mathbb{R}$ satisfying $F(0)=0$ and (7.3) for some nonzero constant $m$, and let $R_{\Phi, \Psi}$ be as in (7.12). Set

$$
m_{0}=m^{-1}\left(\inf _{\|c\|_{2}=1}\left\|c^{T} \Phi\right\|_{2}\right)\left(1-\mu-\mu \frac{\delta\left(V_{2}(\Phi), V_{2}(\Psi)\right)}{\sqrt{1-\left(\delta\left(V_{2}(\Phi), V_{2}(\Psi)\right)^{2}\right.}}\right)
$$

and

$$
L=m^{-1}\left(\sup _{\|c\|_{2}=1}\left\|c^{T} \Phi\right\|_{2}\right) \frac{1+\mu}{\sqrt{1-\left(\delta\left(V_{2}(\Phi), V_{2}(\Psi)\right)\right)^{2}}} .
$$

Then

(i) Given any sampling data $y=\left\langle F\left(x_{\infty}^{T} \Phi\right), \Psi\right\rangle$ of a signal $x_{\infty} \in \ell^{p}$ and any initial guess $x_{0} \in \ell^{p}(\Lambda), 1 \leq p \leq \infty$, the Van-Cittert iteration $x_{n}, n \geq 0$, defined by

$$
x_{n+1}=x_{n}-\alpha R_{\Phi, \Psi}\left(\left\langle F\left(x_{n}^{T} \Phi\right), \Psi\right\rangle-y\right)
$$

converges exponentially to $x_{\infty}$ in $\ell^{p}(\Lambda)$ when the relaxation factor $\alpha$ satisfies $0<\alpha<m_{0} /\left(L+L^{2}\right)$.

(ii) If $F^{\prime \prime}$ is continuous and bounded, then the quasi-Newton iteration defined by

$$
x_{n+1}=x_{n}-\left(R_{\Phi, \Psi}\left\langle\Psi, F^{\prime}\left(x_{n}^{T} \Phi\right) \Phi\right\rangle\right)^{-1} R_{\Phi, \Psi}\left(\left\langle F\left(x_{n}^{T} \Phi\right), \Psi\right\rangle-y\right), \quad n \geq 1,
$$

converges quadratically if the initial guess $x_{0}$ is so chosen that $\|\left\langle F\left(x_{0}^{T} \Phi\right), \Psi\right\rangle-$ $y \|_{p}$ is sufficiently small.

Remark 7.8. In the proof of Theorem 7.7, we show that the Van-Cittert iteration (7.19) converges exponentially for any data $y \in \ell^{p}(\Gamma)$, not necessarily the observed data $\left\langle F\left(x^{T} \Phi\right), \Psi\right\rangle$ of a signal $x \in \ell^{p}(\Lambda)$. Unlike local R-quadratic convergence of the quasi-Newton iteration (7.20) established in Theorem 7.7, the quasi-Newton iteration

$$
x_{n+1}=x_{n}-\alpha_{n}\left(R_{\Phi, \Psi}\left\langle\Psi, F^{\prime}\left(x_{n}^{T} \Phi\right) \Phi\right\rangle\right)^{-1} R_{\Phi, \Psi}\left(\left\langle F\left(x_{n}^{T} \Phi\right), \Psi\right\rangle-y\right), \quad n \geq 1,
$$

with appropriate varying step size $\alpha_{n}$ could have global convergence when $p=2$, see [17]. For $p=2$, we also remark that the following modified Van-Cittert iteration

$$
x_{n+1}=x_{n}-m \tilde{R}\left(\left\langle\Psi, F\left(x_{n}^{T} \Phi\right)\right\rangle-y\right), n \geq 0,
$$

has exponential convergence in $\ell^{2}$ with the assumption (7.3) replaced by a weak condition:

$$
\mu<\sqrt{1-\left(\delta\left(V_{2}(\Phi), V_{2}(\Psi)\right)\right)^{2}}
$$

where $\tilde{R}=\left(A_{\Phi, \Psi}\left(A_{\Psi, \Psi}\right)^{-1} A_{\Psi, \Phi}\right)^{-1} A_{\Phi, \Psi}\left(A_{\Psi, \Psi}\right)^{-1}$. The above exponential convergence can be proved by following the argument in [19]. Without loss of generality, we assume that $\Phi(\operatorname{resp} . \Psi)$ is an orthonormal basis for $V_{2}(\Phi)$ (resp. $V_{2}(\Psi)$ ). Let 
$x_{n}^{1}, x_{n}^{2}, n \geq 0$, be sequences in the Van-Cittert algorithm (7.22) with the observed data $y$ replaced by $y_{1}, y_{2} \in \ell^{2}$. Then it follows from (7.12) and (7.22) that

$$
\begin{aligned}
\left\|x_{n+1}^{1}-x_{n+1}^{2}\right\|_{2} \leq & \left.\| x_{n}^{1}-x_{n}^{2}-m \tilde{R}\left(\left\langle F\left(\left(x_{n}^{1}\right)^{T} \Phi\right), \Psi\right\rangle-\left\langle F\left(\left(x_{n}^{2}\right)^{T} \Phi\right), \Psi\right\rangle\right)\right) \|_{2} \\
& \quad+|m|\left\|y_{1}-y_{2}\right\|_{2} \\
\leq & \left\|\tilde{R}\left\langle\left(x_{n}^{1}-x_{n}^{2}\right)^{T} \Phi-m F\left(\left(x_{n}^{1}\right)^{T} \Phi\right)+m F\left(\left(x_{n}^{2}\right)^{T} \Phi\right), \Psi\right\rangle\right\|_{2} \\
& \quad+|m|\left\|y_{1}-y_{2}\right\|_{2} \\
\leq & |m|\left\|y_{1}-y_{2}\right\|_{2}+\left(1-\left(\delta\left(V_{2}(\Phi), V_{2}(\Psi)\right)\right)^{2}\right)^{-1 / 2} \\
& \quad \times\left\|\left(x_{n}^{1}-x_{n}^{2}\right)^{T} \Phi-m F\left(\left(x_{n}^{1}\right)^{T} \Phi\right)+m F\left(\left(x_{n}^{2}\right)^{T} \Phi\right)\right\|_{2} \\
\leq & \left(1-\left(\delta\left(V_{2}(\Phi), V_{2}(\Psi)\right)\right)^{2}\right)^{-1 / 2} \mu\left\|x_{n}^{1}-x_{n}^{2}\right\|_{2}+|m|\left\|y_{1}-y_{2}\right\|_{2}
\end{aligned}
$$

for all $n \geq 0$. Taking $y_{2}=y_{1}=y \in \ell^{2}$ in (7.24) and using (7.23) shows that the map $V(x):=x-m \tilde{R}\left(\left\langle\Psi, F\left(x^{T} \Phi\right)\right\rangle-y\right)$ is contractive on $\ell^{2}$ and hence has a unique fixed point $V^{-1}(y)$. Applying (7.24) with $y_{1}=y_{2}=y$ and $x_{0}^{2}=V^{-1}(y)$ leads to the exponential convergence of the Van-Cittert algorithm (7.22),

$$
\left\|x_{n}-V^{-1}(y)\right\|_{2} \leq \mu^{n}\left(1-\left(\delta\left(V_{2}(\Phi), V_{2}(\Psi)\right)\right)^{2}\right)^{-n / 2}\left\|x_{0}-V^{-1}(y)\right\|_{2}, \quad n \geq 0 .
$$

Also taking limit in (7.24) shows that the Van-Cittert algorithm (7.22) has $\ell^{2}$ stability:

$$
\left\|V^{-1}\left(y_{1}\right)-V^{-1}\left(y_{2}\right)\right\|_{2} \leq \frac{\sqrt{1-\left(\delta\left(V_{2}(\Phi), V_{2}(\Psi)\right)\right)^{2}}}{\sqrt{1-\left(\delta\left(V_{2}(\Phi), V_{2}(\Psi)\right)\right)^{2}}-\mu}\left\|y_{1}-y_{2}\right\|_{2}
$$

for any $y_{1}, y_{2} \in \ell^{2}$. From the above argument, we see that the limit $V^{-1}(y)$ of the Van-Cittert algorithm (7.22) satisfies $\tilde{R}\left(\left\langle\Psi, F\left(\left(T^{-1}(y)\right)^{T} \Phi\right)\right\rangle-y\right)=0$. The above consistence condition is established in [19] under additional assumption that $\tilde{R}$ is invertible.

Proof of Theorem 7.7. (i) Let $g_{F, \Phi, \Psi}$ be the function on $\ell^{p}(\Lambda), 1 \leq p \leq \infty$, defined in (17.13). By the argument in the proof of Theorem 17.1, the function $g_{F, \Phi, \Psi}$ satisfies all requirements in Theorem 6.1. Then applying Theorem 6.1 gives the exponential convergence of the sequence $x_{n}, n \geq 0$, in the Van-Cittert iteration (7.19), and the limit follows from the invertibility of the function $g_{F, \Phi, \Psi}$ in $\ell^{p}$.

(ii) By (7.12) and (7.14), we obtain

$$
\begin{aligned}
& \left\|\nabla g_{F, \Phi, \Psi}(x)-\nabla g_{F, \Phi, \Psi}(\tilde{x})\right\|_{\mathcal{J}_{\beta}(\Lambda)} \\
\leq & C\left\|\left\langle\Psi,\left(F^{\prime}\left(x^{T} \Phi\right)-F^{\prime}\left(\tilde{x}^{T} \Phi\right)\right) \Phi\right\rangle\right\|_{\mathcal{J}_{\beta}(\Gamma, \Lambda)} \\
\leq & C\left\|F^{\prime \prime}\right\|_{\infty}\left\|x^{T} \Phi-\tilde{x}^{T} \Phi\right\|_{\infty} \leq C\|x-\tilde{x}\|_{\infty} \leq C\|x-\tilde{x}\|_{p}
\end{aligned}
$$

for all $x, \tilde{x} \in \ell^{p}$. Then the local R-quadratic convergence of the quasi-Newton iteration (7.20) follows from Theorem 6.4.

7.3. Numerical simulation. In this subsection, we present some numerical simulations for demonstration of the theoretical results in Theorem 7.7. Let $\Lambda=$ $\left\{t_{i}\right\}_{i=1}^{40}$ contain randomly selected knots $t_{i} \in[-2,2], 1 \leq i \leq 40$, satisfying $0.05 \leq$ $\min _{1 \leq i \leq 39} t_{i+1}-t_{i} \leq \max _{1 \leq i \leq 39} t_{i+1}-t_{i} \leq 0.15, \Gamma=\{-2+i / 20\}_{i=1}^{80}$ be the set 
of uniform knots on $[-2,2], \Phi=\left(\phi_{t_{i}}\right)_{t_{i} \in \Lambda}$ be the column vector of interpolating cubic splines with knots $\Lambda$ satisfying $\phi_{t_{i}}\left(t_{j}\right)=\delta_{i j}$ for all $1 \leq i, j \leq 40$ where $\delta_{i j}$ is the Kronecker symbol, $\Psi=\left\{10 \chi_{[-2+(i-1) / 20,-2+i / 20)}\right\}_{i=1}^{80}$ be the column vector of uniform sampling functionals on $\Gamma$, and $F(t)=\sin (\pi t / 2)$ be the companding function. In the simulation we use $x_{\infty}=\left(c_{1}, \ldots, c_{40}\right)^{T} / \max _{1 \leq i \leq 40}\left|c_{i}\right|$ as the original sequence to be recovered from its nonlinear samples $\left\langle F\left(x_{\infty}^{T} \Phi\right), \Psi\right\rangle$ (plotted in Figure 2(b)), where $c_{i} \in[-1 / 2,1 / 2], 1 \leq i \leq 40$. The nonuniform cubic spline $x_{\infty}^{T} \Phi$ is plotted in Figure 2(a) in a continuous line. We add piecewise random noise $\epsilon=(r(1) a(1), \ldots, r(80) a(80))^{T}\left\|\left\langle F\left(x_{\infty}^{T} \Phi\right), \Psi\right\rangle\right\|_{\infty}$ of noise level from $0 \%$ to $2.5 \%$, plotted in Figure 2(b) in a dashed line, where $r(i) \in[-0.05,0.05], 1 \leq i \leq 80$, are randomly selected and $a(i), 1 \leq i \leq 80$, take value one when $i \in[1,16) \cup[72,80]$, two when $i \in[24,40) \cup[48,64)$, and zero otherwise. We reconstruct the signal $x_{\infty}^{\epsilon}$ from the noisy nonlinear samples $\left\langle F\left(x_{\infty}^{T} \Phi\right), \Psi\right\rangle+\epsilon$ by applying the Van-Cittert iterative method (7.19) with $x_{0}=0$ and $y=\left\langle F\left(x_{\infty}^{T} \Phi\right), \Psi\right\rangle+\epsilon$, and plot the difference between cubic splines $\left(x_{\infty}^{\epsilon}\right)^{T} \Phi$ and $x_{\infty}^{T} \Phi$ in Figure 2(a) in a dashed line. The numerical result shows that $\left\|x_{\infty}^{\epsilon}-x_{\infty}\right\|_{\infty}=0.003$, which illustrates the $\ell^{\infty}$-stability of the reconstruction procedure in Theorem [7.7. The nonlinear sampling procedure $S: x \longmapsto\left\langle F\left(x^{T} \Phi\right), \Psi\right\rangle$ is locally behaved as entries in the column vectors $\Phi$ and $\Psi$ are locally supported, which can be observed by comparing the shape of the original cubic spline $x_{\infty}^{T} \Phi$ in Figure 2(a) and the nonlinear samples $\left\langle F\left(x^{T} \Phi\right), \Psi\right\rangle$ in Figure 2(b). For the sampling procedure without instantaneous companding (i.e. $F(t)=t$ ), it is shown in [7, 59] that the reconstruction procedure is also locally behaved, that is, the amplitude of a signal at any position is essentially determined by the adjacent sampling values. We notice that the cubic splines $\left(x_{\infty}^{\epsilon}\right)^{T} \Phi$ and $x_{\infty}^{T} \Phi$ are almost perfectly matched in the interval $[0,0.4]$ where no noise is added to adjacent nonlinear samples, which indicates that the reconstruction procedure from the nonlinear samples is locally behaved.

We apply the Van-Cittert iterative method (7.19) with zero initial guess $x_{0}=0$ and relaxation factor $\alpha=0.3$ to recover the signal $x_{\infty}$ from its nonlinear samples $\left\langle F\left(x_{\infty}^{T} \Phi\right), \Psi\right\rangle$. The corresponding numerical error is presented in Table 1, where listed in the first column is the number of iteration, listed in the second, third and fourth columns are the $\ell^{\infty}$-error $\left\|x_{n}-x_{\infty}\right\|_{\infty}$, the mean squared error $\left\|x_{n}-x_{\infty}\right\|_{2}$, and the $\ell^{1}$-error $\left\|x_{n}-x_{\infty}\right\|_{1}$ respectively, and listed in the last column is the $\ell^{\infty}$-error $\left\|\left\langle F\left(x_{n}^{T} \Phi\right), \Psi\right\rangle-\left\langle F\left(x_{\infty}^{T} \Phi\right), \Psi\right\rangle\right\|_{\infty}$ of nonlinear sample data.

A difficult problem is how to select the relaxation factor $\alpha$. We tried to change the relaxation factor in every iteration, c.f. (7.21). The ideas are to use smaller relaxation factor if the $\ell^{\infty}$-error $\left\|\left\langle F\left(x_{n}^{T} \Phi\right), \Psi\right\rangle-y\right\|_{\infty}$ between the nonlinear sample data of the $n$-th iteration $x_{n}$ and the given nonlinear sample data $y$ increases, and to use larger relaxation factor when the ratio between $\left\|\left\langle F\left(x_{n}^{T} \Phi\right), \Psi\right\rangle-y\right\|_{\infty}$ and $\|y\|_{\infty}$ gets smaller. The above modification of the Van-Cittert iteration method does provide faster convergence, but no significant improvement is observed. We also apply the combination of the Van-Cittert iteration method and the quasi-Newton iteration method. As shown in Theorem 17.7, the Van-Cittert iteration method has slow global convergence and the quasi-Newton iteration method has fast local 


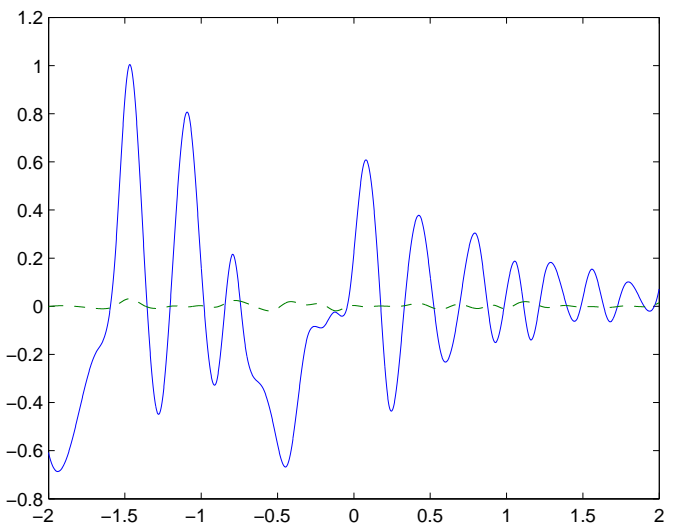

(a)

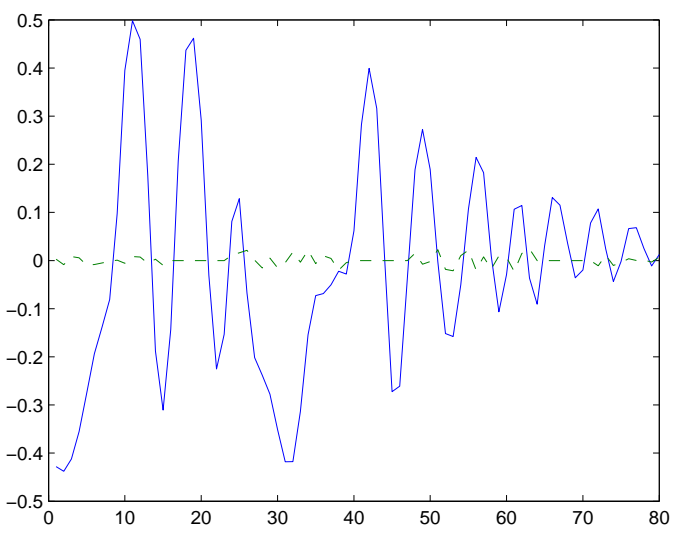

(b)

Figure 2. (a) The cubic spline $x_{\infty}^{T} \Phi$ is plotted in a continuous line, while it is plotted in a dashed line the difference between the original cubic spline $x_{\infty}^{T} \Phi$ and the reconstructed cubic spline $x_{\infty}^{\epsilon} \Phi$ from noisy nonlinear sampled data $\left\langle F\left(x_{\infty}^{T} \Phi\right), \Psi\right\rangle+\epsilon$. (b) Nonlinear sampled data $\left\langle F\left(x_{\infty}^{T} \Phi\right), \Psi\right\rangle$ is plotted in a continuous line, and the piecewise random noise $\epsilon$ with noise level between $0 \%$ and $2.5 \%$ is in a dashed line.

convergence. So we may use the Van-Cittert iteration at the beginning and the quasi-Newton iteration when the ratio between $\left\|\left\langle F\left(x_{n}^{T} \Phi\right), \Psi\right\rangle-y\right\|_{\infty}$ and $\|y\|_{\infty}$ is below certain threshold, say $10 \%$. The numerical error is shown in Table2, where the quasi-Newton iteration method (7.20) is used from the 6-th iteration. Comparing the numerical results in Table 1 and Table 2, we may conclude that, in order to recover a signal from its nonlinear sample data, it is much better to use the combination of the Van-Cittert iteration method and the quasi-Newton iteration method than to use the Van-Cittert iteration method solely. 
TABLE 1. Reconstruction error via the Van-Cittert iteration

\begin{tabular}{ccccc}
\hline \hline Iteration & $\ell^{\infty}$-error & $\frac{\ell^{2}-\text { error }}{\sqrt{40}}$ & $\frac{\ell^{1}-\text { error }}{40}$ & $\ell^{\infty}$-data \\
\hline 5 & 1.9893 & 0.5049 & 0.3334 & 0.1168 \\
10 & 0.5230 & 0.1832 & 0.1533 & 0.0425 \\
15 & 0.2102 & 0.0931 & 0.0846 & 0.0222 \\
20 & 0.1053 & 0.0535 & 0.0503 & 0.0128 \\
30 & 0.0343 & 0.0201 & 0.0194 & 0.0048 \\
40 & 0.0128 & 0.0081 & 0.0078 & 0.0019 \\
50 & 0.0050 & 0.0033 & 0.0032 & 0.0008 \\
\hline \hline
\end{tabular}

TABLE 2. Reconstruction error via a combination of the Van-Cittert iteration and the quasi-Newton iteration

\begin{tabular}{ccccc}
\hline \hline Iteration & $\ell^{\infty}$-error & $\frac{\ell^{2}-\text { error }}{\sqrt{40}}$ & $\frac{\ell^{1}-\text { error }}{40}$ & $\ell^{\infty}$-data \\
\hline 5 & 1.9893 & 0.5049 & 0.3334 & 0.1168 \\
6 & 0.2372 & 0.1175 & 0.1073 & 0.0896 \\
7 & 0.2034 & 0.1033 & 0.0954 & 0.0250 \\
8 & 0.0232 & 0.0170 & 0.0168 & 0.0222 \\
9 & 0.0010 & 0.0008 & 0.0007 & 0.0037 \\
10 & 0.0000 & 0.0000 & 0.0000 & 0.0002 \\
\hline \hline
\end{tabular}

\section{LOCAL IDENTIFICATION OF INNOVATION POSITIONS AND QUALIFICATION OF} AMPLITUDES

In this section, we apply the theory established for the nonlinear functional equation (1.1) with $p=\infty$ in the first two parts of this paper indirectly to local identification of innovation positions and qualification of amplitudes of a signal $h$ having the parametric representation (1.13). In the first subsection, we show that innovation positions and amplitudes of a signal $h$ having the parametric representation (1.13) can be precisely identified and qualified provided that the linearization at approximate innovation positions and amplitudes is stable, see Theorem 8.1. In the second subsection, we show that any signal $h$ in a perturbed shift-invariant space with unknown (but small) perturbations can be recovered exactly from its average samples $\left\langle h, \psi_{m}(\cdot-k)\right\rangle, 1 \leq m \leq M, k \in \mathbb{Z}^{d}$, provided that the generator $\varphi$ of the perturbed shift-invariant space and the average samplers $\psi_{m}, 1 \leq m \leq M$, satisfy (1.21). In the last subsection, we present some numerical simulations to demonstrate the precise identification and accurate qualification.

\subsection{Precise identification of innovation positions and accurate qualifica- tion of amplitudes.}

Theorem 8.1. Let $\Lambda_{0}$ and $\Gamma$ be relatively-separated subsets of $\mathbb{R}^{d}, c_{0}:=\left(c_{0}(\lambda)\right)_{\lambda \in \Lambda_{0}} \in$ $\ell^{\infty}\left(\Lambda_{0}\right), \Psi=\left(\psi_{\gamma}\right)_{\gamma \in \Gamma}$ have polynomial decay of order $\beta>d$, the impulse response 
$\varphi \in C^{2}\left(\mathbb{R}^{d}\right)$ satisfy

$$
\left\|\varphi(\cdot)(1+|\cdot|)^{\beta}\right\|_{\infty}+\left\|\nabla \varphi(\cdot)(1+|\cdot|)^{\beta}\right\|_{\infty}+\left\|\nabla^{2} \varphi(\cdot)(1+|\cdot|)^{\beta}\right\|_{\infty}<\infty,
$$

and let the companding function $F \in C^{2}(\mathbb{R})$ satisfy

$$
\left\|F^{\prime}\right\|_{\infty}+\left\|F^{\prime \prime}\right\|_{\infty}<\infty .
$$

If the nonlinear sampling process (1.15) has its linearization at $\left(\Lambda_{0}, c_{0}\right)$ being stable (i.e., the matrix $S_{\Lambda_{0}, c_{0}}$ in (1.17) satisfies (1.16)), then there exists a positive constant $\delta_{0}$ such that any signal $h$, that has the parametric representation $h(t)=$ $\sum_{\lambda \in \Lambda_{0}}\left(c_{0}(\lambda)+c(\lambda)\right) \varphi(t-\lambda-\sigma(\lambda))$ for some vectors $c:=(c(\lambda))_{\lambda \in \Lambda_{0}}$ and $\sigma:=$ $(\sigma(\lambda))_{\lambda \in \Lambda_{0}}$ with $\max \left(\|c\|_{\infty},\|\sigma\|_{\infty}\right) \leq \delta_{0}$, can be stably recovered from its sampling data $\langle F(h), \Psi\rangle$.

Proof. To prove the desired stable recovery, we need to find a small number $\delta_{0}$ and two positive constants $C_{1}$ and $C_{2}$ such that

$$
\begin{aligned}
C_{1}\left(\left\|c_{1}-c_{2}\right\|_{\infty}+\left\|\sigma_{1}-\sigma_{2}\right\|_{\infty}\right) & \leq\left\|\left\langle F\left(h_{1}\right)-F\left(h_{2}\right), \Psi\right\rangle\right\|_{\infty} \\
& \leq C_{2}\left(\left\|c_{1}-c_{2}\right\|_{\infty}+\left\|\sigma_{1}-\sigma_{2}\right\|_{\infty}\right)
\end{aligned}
$$

for all vectors $c_{i}:=\left(c_{i}(\lambda)\right)_{\lambda \in \Lambda_{0}}$ and $\sigma_{i}:=\left(\sigma_{i}(\lambda)\right)_{\lambda \in \Lambda_{0}}$ with $\max \left(\left\|c_{i}\right\|_{\infty},\left\|\sigma_{i}\right\|_{\infty}\right) \leq \delta_{0}$, where $h_{i}=\sum_{\lambda \in \Lambda_{0}}\left(c_{0}(\lambda)+c_{i}(\lambda)\right) \varphi\left(\cdot-\lambda-\sigma_{i}(\lambda)\right), i=1,2$.

By (8.2) and the polynomial decay property for the average sampler $\Psi$, we obtain

$$
\begin{aligned}
& \left\|\left\langle F\left(h_{1}\right)-F\left(h_{2}\right), \Psi\right\rangle\right\|_{\infty} \\
\leq & C\left\|F^{\prime}\right\|_{\infty}\|\Psi\|_{\infty, \beta}\left\|c_{0}+c_{2}\right\|_{\infty}\left\|\sum_{\lambda \in \Lambda_{0}} \mid \varphi\left(\cdot-\lambda-\sigma_{1}(\lambda)\right)-\varphi\left(\cdot-\lambda-\sigma_{2}(\lambda)\right)\right\|_{\infty} \\
& +C\left\|F^{\prime}\right\|_{\infty}\|\Psi\|_{\infty, \beta}\left\|c_{1}-c_{2}\right\|_{\infty}\left\|\sum_{\lambda \in \Lambda_{0}}\left|\varphi\left(\cdot-\lambda-\sigma_{1}(\lambda)\right)\right|\right\|_{\infty} \\
\leq & C\left(1+\delta_{0}\right)^{\beta}\left(\left\|c_{0}\right\|_{\infty}+\delta_{0}\right)\left\|F^{\prime}\right\|_{\infty}\|\Psi\|_{\infty, \beta}\left\|\nabla \varphi(\cdot)(1+|\cdot|)^{\beta}\right\|_{\infty}\left\|\sigma_{1}-\sigma_{2}\right\|_{\infty} \\
& +C\left(1+\delta_{0}\right)^{\beta}\left\|F^{\prime}\right\|_{\infty}\|\Psi\|_{\infty, \beta}\left\|\varphi(\cdot)(1+|\cdot|)^{\beta}\right\|_{\infty}\left\|c_{1}-c_{2}\right\|_{\infty} .
\end{aligned}
$$

This proves the second inequality in (8.3).

Now we prove the first inequality in (8.3). From (8.1), (8.2) and the polynomial decay property for the average sampler $\Psi$, it follows that

$$
\begin{aligned}
& \left|\left\langle F^{\prime}(h) \varphi(\cdot-\lambda-\sigma(\lambda)), \psi_{\gamma}\right\rangle\right|+\left|\left\langle F^{\prime}(h) \nabla \varphi(\cdot-\lambda-\sigma(\lambda)), \psi_{\gamma}\right\rangle\right| \\
\leq \quad & C\left\|F^{\prime}\right\|_{\infty}\left(\left\|\varphi(\cdot)(1+|\cdot|)^{\beta}\right\|_{\infty}+\left\|\nabla \varphi(\cdot)(1+|\cdot|)^{\beta}\right\|_{\infty}\right)\|\Psi\|_{\infty, \beta}\left(1+\|\sigma\|_{\infty}\right)^{\beta} \\
& \times(1+|\gamma-\lambda|)^{-\beta} \quad \text { for all } \gamma \in \Gamma \text { and } \lambda \in \Lambda_{0} .
\end{aligned}
$$

Hence the matrix

$$
A\left(\begin{array}{l}
\sigma \\
c
\end{array}\right):=\left(\begin{array}{c}
-\left(c_{0}(\lambda)+c(\lambda)\right)\left\langle F^{\prime}(h) \nabla \varphi(\cdot-\lambda-\sigma(\lambda)), \psi_{\gamma}\right\rangle \\
\left\langle F^{\prime}(h) \varphi(\cdot-\lambda-\sigma(\lambda)), \psi_{\gamma}\right\rangle
\end{array}\right)_{\gamma \in \Gamma, \lambda \in \Lambda_{0}}
$$

is well-defined for any $c:=(c(\lambda))_{\lambda \in \Lambda_{0}} \in \ell^{\infty}\left(\Lambda_{0}\right)$ and $\sigma:=(\sigma(\lambda))_{\lambda \in \Lambda_{0}} \in\left(\ell^{\infty}\left(\Lambda_{0}\right)\right)^{d}$, where $h=\sum_{\lambda \in \Lambda_{0}}\left(c_{0}(\lambda)+c(\lambda)\right) \varphi(\cdot-\lambda-\sigma(\lambda))$. Moreover $\left(A\left(\begin{array}{l}\sigma \\ c\end{array}\right)\right)^{T} A\left(\begin{array}{l}\sigma \\ c\end{array}\right)$ belongs to 
the Jaffard class $\left(\mathcal{J}_{\beta}\left(\Lambda_{0}\right)\right)^{d+1}$, and

$$
\left\|\left(A\left(\begin{array}{c}
\sigma \\
c
\end{array}\right)\right)^{T} A\left(\begin{array}{l}
\sigma \\
c
\end{array}\right)\right\|_{\left(\mathcal{J}_{\beta}\left(\Lambda_{0}\right)\right)^{d+1}} \leq C\left(1+\|\sigma\|_{\infty}\right)^{2 \beta}\left(1+\|c\|_{\infty}\right)^{2}
$$

by (8.4) and the relatively-separatedness of the sets $\Lambda_{0}$ and $\Gamma$. Notice that $A\left(\begin{array}{l}\sigma \\ c\end{array}\right)$ becomes the matrix $S_{\Lambda_{0}, c_{0}}$ in (1.17) when $\sigma=0$ and $c=0$. Then $\left(S_{\Lambda_{0}, c_{0}}\right)^{T} S_{\Lambda_{0}, c_{0}}$ belongs to the Jaffard class $\left(\mathcal{J}_{\beta}\left(\Lambda_{0}\right)\right)^{d+1}$ by (8.5). This, together with the stability condition (1.16) and the Wiener's lemma for infinite matrices in the Jaffard class [32, 58], proves that $\left(\left(S_{\Lambda_{0}, c_{0}}\right)^{T} S_{\Lambda_{0}, c_{0}}\right)^{-1}$ belongs to the Jaffard class $\left(\mathcal{J}_{\beta}\left(\Lambda_{0}\right)\right)^{d+1}$; i.e.,

$$
\left\|\left(\left(S_{\Lambda_{0}, c_{0}}\right)^{T} S_{\Lambda_{0}, c_{0}}\right)^{-1}\right\|_{\left(\mathcal{J}_{\beta}\left(\Lambda_{0}\right)\right)^{d+1}} \leq D_{1}
$$

for some positive constant $D_{1}$. Using similar argument to prove (8.4), we get

$$
\begin{aligned}
& \left|\left\langle F^{\prime}(h) \varphi(\cdot-\lambda-\sigma(\lambda)), \psi_{\gamma}\right\rangle-\left\langle F^{\prime}\left(h_{0}\right) \varphi(\cdot-\lambda), \psi_{\gamma}\right\rangle\right| \\
& +\left|\left\langle F^{\prime}(h) \nabla \varphi(\cdot-\lambda-\sigma(\lambda)), \psi_{\gamma}\right\rangle-\left\langle F^{\prime}\left(h_{0}\right) \nabla \varphi(\cdot-\lambda), \psi_{\gamma}\right\rangle\right| \\
\leq & C\left(1+\|\sigma\|_{\infty}\right)^{\beta}\left(\|\sigma\|_{\infty}+\|c\|_{\infty}\right)(1+|\lambda-\gamma|)^{-\beta}
\end{aligned}
$$

for all $\lambda \in \Lambda_{0}$ and $\gamma \in \Gamma$. Therefore

$$
\begin{aligned}
& \left\|\left(\left(S_{\Lambda_{0}, c_{0}}\right)^{T} S_{\Lambda_{0}, c_{0}}\right)^{-1} S_{\Lambda_{0}, c_{0}}^{T} A\left(\begin{array}{c}
\sigma \\
c
\end{array}\right)-I\right\|_{\left(\mathcal{J}_{\beta}\left(\Lambda_{0}\right)\right)^{d+1}} \\
\leq & C\left(1+\|\sigma\|_{\infty}\right)^{\beta}\left(1+\|c\|_{\infty}\right)\left(\|c\|_{\infty}+\|\sigma\|_{\infty}\right),
\end{aligned}
$$

where $I$ is the identity matrix of appropriate size. For the function $f_{\Lambda_{0}, c_{0}, \Psi}$ in (1.18), we notice that

$$
\nabla f_{\Lambda_{0}, c_{0}, \Psi}\left(\begin{array}{c}
\sigma \\
c
\end{array}\right)=\left(\left(S_{\Lambda_{0}, c_{0}}\right)^{T} S_{\Lambda_{0}, c_{0}}\right)^{-1} S_{\Lambda_{0}, c_{0}}^{T} A\left(\begin{array}{l}
\sigma \\
c
\end{array}\right)
$$

Then $\nabla f_{\Lambda_{0}, c_{0}, \Psi}\left(\begin{array}{c}\sigma \\ c\end{array}\right)$ belongs to the Jaffard class $\left(\mathcal{J}_{\beta}\left(\Lambda_{0}\right)\right)^{d+1}$ and

$$
\left\|\nabla f_{\Lambda_{0}, c_{0}, \Psi}\left(\begin{array}{l}
\sigma \\
c
\end{array}\right)-I\right\|_{\left(\mathcal{J}_{\beta}\left(\Lambda_{0}\right)\right)^{d+1}} \leq C(1+\|\sigma\|)^{\beta}\left(1+\|c\|_{\infty}\right)\left(\|c\|_{\infty}+\|\sigma\|_{\infty}\right)
$$

by (8.8). Recall that any infinite matrix in the Jaffard class $\left(\mathcal{J}_{\beta}\left(\Lambda_{0}\right)\right)^{d+1}$ is a bounded operator on $\left(\ell^{\infty}(\Lambda)\right)^{d+1}$. Thus there exists a positive number $\delta_{0}$ such that

$$
\left\|\nabla f_{\Lambda_{0}, c_{0}, \Psi}\left(\begin{array}{l}
\sigma \\
c
\end{array}\right)-I\right\|_{\mathcal{B}\left(\left(\ell^{\infty}\left(\Lambda_{0}\right)\right)^{d+1}\right)} \leq \frac{1}{3}
$$

for vectors $\sigma \in\left(\ell^{\infty}\left(\Lambda_{0}\right)\right)^{d}$ and $c \in \ell^{\infty}\left(\Lambda_{0}\right)$ with $\max \left(\|c\|_{\infty},\|\sigma\|_{\infty}\right) \leq \delta_{0}$. This implies that there exists a positive constant $\delta_{0}$ such that

$$
\begin{aligned}
\frac{2}{3} \max \left(\left\|\sigma_{1}-\sigma_{2}\right\|_{\infty},\left\|c_{1}-c_{2}\right\|_{\infty}\right) & \leq\left\|f_{\Lambda_{0}, c_{0}, \Psi}\left(\begin{array}{l}
\sigma_{1} \\
c_{1}
\end{array}\right)-f_{\Lambda_{0}, c_{0}, \Psi}\left(\begin{array}{l}
\sigma_{2} \\
c_{2}
\end{array}\right)\right\|_{\infty} \\
& \leq \frac{4}{3} \max \left(\left\|\sigma_{1}-\sigma_{2}\right\|_{\infty},\left\|c_{1}-c_{2}\right\|_{\infty}\right)
\end{aligned}
$$

for vectors $\sigma_{i} \in\left(\ell^{\infty}\left(\Lambda_{0}\right)\right)^{d}$ and $c_{i} \in \ell^{\infty}\left(\Lambda_{0}\right)$ with $\max \left(\left\|c_{i}\right\|_{\infty},\left\|\sigma_{i}\right\|_{\infty}\right) \leq \delta_{0}, i=1,2$. The first inequality in (8.3) then follows from (8.4), (8.6) and (8.11). 
Theorem 8.2. Let $\varphi, \Psi, F, \Lambda_{0}, c_{0}, \delta_{0}$ be as in Theorem 8.1, and $f_{\Lambda_{0}, c_{0}, \Psi}$ as in (1.18). Given the sampling data $y=\langle F(h), \Psi\rangle$ of a signal $h=\sum_{\lambda \in \Lambda_{0}}\left(c_{0}(\lambda)+c(\lambda)\right) \varphi(\cdot-\lambda-$ $\sigma(\lambda))$ with $c=(c(\lambda))_{\lambda \in \Lambda_{0}}$ and $\sigma=(\sigma(\lambda))_{\lambda \in \Lambda_{0}}$ satisfying $\max \left(\|c\|_{\infty},\|\sigma\|_{\infty}\right) \leq \delta_{0} / 2$, we define the Van-Cittert iteration by

$$
\left(\begin{array}{c}
\sigma_{n+1} \\
d_{n+1}
\end{array}\right)=\left(\begin{array}{c}
\sigma_{n} \\
d_{n}
\end{array}\right)-\alpha\left(f_{\Lambda_{0}, c_{0}, \Psi}\left(\begin{array}{c}
\sigma_{n} \\
d_{n}
\end{array}\right)-z_{0}\right), \quad n \geq 0
$$

with $\left(\begin{array}{c}\sigma_{0} \\ d_{0}\end{array}\right)=0, \alpha \in(0,1)$, and $\left.z_{0}=\left(S_{\Lambda_{0}, c_{0}}\right)^{T} S_{\Lambda_{0}, c_{0}}\right)^{-1} S_{\Lambda_{0}, c_{0}}^{T}\left(y-\left\langle F\left(h_{0}\right), \Psi\right\rangle\right)$. Then $\left(\begin{array}{l}\sigma_{n} \\ d_{n}\end{array}\right), n \geq 0$, converges to the solution $\left(\begin{array}{l}\sigma \\ c\end{array}\right)$ exponentially.

Proof. By (8.10), (8.11) and (8.12), we have

$$
\max \left(\left\|\sigma_{1}\right\|_{\infty},\left\|d_{1}\right\|_{\infty}\right) \leq \frac{4 \alpha}{3} \max \left(\|\sigma\|_{\infty},\|c\|_{\infty}\right)
$$

and

$$
\max \left(\left\|\sigma_{n+1}-\sigma_{n}\right\|_{\infty},\left\|d_{n+1}-d_{n}\right\|_{\infty}\right) \leq \frac{3-2 \alpha}{3} \max \left(\left\|\sigma_{n}-\sigma_{n-1}\right\|_{\infty},\left\|d_{n}-d_{n-1}\right\|_{\infty}\right)
$$

for all $n \geq 1$. Hence $\max \left(\left\|\sigma_{n}\right\|_{\infty},\left\|d_{n}\right\|_{\infty}\right) \leq \delta_{0}$ and the sequence $\left(\begin{array}{c}\sigma_{n} \\ d_{n}\end{array}\right), n \geq 0$, in the Van-Cittert iteration ( $(8.12)$ converges to the solution $\left(\begin{array}{l}\sigma \\ c\end{array}\right)$ exponentially.

\subsection{Sampling in a perturbed shift-invariant space with unknown pertur- bation.}

Theorem 8.3. Let $\varphi$ satisfy (8.1) and $\psi_{1}, \ldots, \psi_{M}$ satisfy

$$
\sum_{1 \leq m \leq M}\left\|\psi_{m}(\cdot)(1+|\cdot|)^{\beta}\right\|_{\infty}<\infty
$$

If (1.21) holds, then for any $L \geq 1$ there exists a positive number $\delta_{1} \in(0,1 / 2)$ such that any signal $h(t)=\sum_{k \in \mathbb{Z}^{d}} c(k) \varphi(t-k-\sigma(k))$ with $\left\|(\sigma(k))_{k \in \mathbb{Z}^{d}}\right\|_{\infty} \leq \delta_{1}$ and $\left\|(c(k))_{k \in \mathbb{Z}^{d}}\right\|_{\ell_{\varnothing}^{\infty}}:=\sup _{c(k) \neq 0}|c(k)|+|c(k)|^{-1} \leq L$ can be recovered from its average sample data $\left\langle h, \psi_{m}(\cdot-k)\right\rangle, 1 \leq m \leq M, k \in \mathbb{Z}^{d}$, in a stable way.

Remark 8.4. Let $\varphi$ and $\psi_{1}, \ldots, \psi_{M}$ be as in Theorem 8.3, and let

$V_{2}(\varphi, \nabla \varphi)=\left\{\left.\sum_{k \in \mathbb{Z}^{d}}\left(e_{o}(k) \varphi(\cdot-k)+\left(e_{g}(k)\right)^{T} \nabla \varphi(\cdot-k)\right)\left|\sum_{k \in \mathbb{Z}^{d}}\right| e_{0}(k)\right|^{2}+\left|e_{g}(k)\right|^{2}<\infty\right\}$

be the shift-invariant space generated by $\varphi$ and its first-order partial derivatives. Then an equivalent formulation of the condition (1.21) is that the integer-shifts of $\varphi$ and its first-order partial derivatives form a Riesz basis for the space $V_{2}(\varphi, \nabla \varphi)$ and signals $h$ living in $V_{2}(\varphi, \nabla \varphi)$ can be recovered stably from their samples $\left\langle h, \psi_{m}(\cdot-\right.$ $k)\rangle, 1 \leq m \leq M, k \in \mathbb{Z}^{d}$. The readers may refer to [2, 3, 62, 65] and references therein for sampling and reconstruction of signals in a shift-invariant space. 
Proof of Theorem 8.3. By (1.21), the vector $A(\xi):=\left(\left[\widehat{\varphi}, \widehat{\psi}_{1}\right](\xi), \cdots,\left(\left[\widehat{\varphi}, \widehat{\psi}_{M}\right](\xi)\right)\right.$ is nonzero for all $\xi \in \mathbb{R}^{d}$. Here the bracket product is defined as follows: $[f, g](\xi)=$ $\sum_{l \in \mathbb{Z}^{d}} f(\xi+2 l \pi) \overline{g(\xi+2 l \pi)}$. Hence

$$
R(\xi):=\sum_{m=1}^{M}\left|\left[\widehat{\varphi}, \widehat{\psi}_{m}\right](\xi)\right|^{2}>0 \quad \text { for all } \xi \in \mathbb{R}^{d} .
$$

By (8.1) and (8.13), the Fourier coefficients of $\left[\widehat{\varphi}, \widehat{\psi}_{m}\right](\xi), 1 \leq m \leq M$, have polynomial decay of order $\beta>d$. Here a periodic function $f(\xi)=\sum_{k \in \mathbb{Z}^{d}} c_{f}(k) e^{-i k \xi}$ is said to have Fourier coefficients with polynomial decay of order $\beta \geq 0$ if $\sup _{k \in \mathbb{Z}^{d}} \mid c_{f}(k)(1+$ $|k|)^{\beta}<\infty$. This, together with (8.14) and Wiener's lemma for infinite matrices in the Jaffard class [32, 58], implies that $(R(\xi))^{-1}$ has Fourier coefficients with polynomial decay of order $\beta>d$. So does

$$
(R(\xi))^{-1} A(\xi):=\left(\sum_{k \in \mathbb{Z}^{d}} r_{1}(k) e^{-i k \xi}, \cdots, \sum_{k \in \mathbb{Z}^{d}} r_{M}(k) e^{-i k \xi}\right) .
$$

Thus

$$
D_{2}:=\sup _{1 \leq m \leq M} \sum_{k \in \mathbb{Z}^{d}}\left|r_{m}(k)\right|<\infty
$$

Given the sampling data $y_{m}(k):=\left\langle h, \psi_{m}(\cdot-k)\right\rangle, 1 \leq m \leq M, k \in \mathbb{Z}^{d}$, of a signal $h(t)=\sum_{k \in \mathbb{Z}^{d}} c(k) \varphi(\cdot-k-\sigma(k))$ satisfying $\left\|(c(k))_{k \in \mathbb{Z}^{d}}\right\|_{\ell_{\varnothing}^{\infty}} \leq L$ and $\left\|(\sigma(k))_{k \in \mathbb{Z}^{d}}\right\|_{\infty} \leq$ $\delta_{1}$. Define $\tilde{c}:=(\tilde{c}(k))_{k \in \mathbb{Z}^{d}}$ by $\tilde{c}(k)=\sum_{m=1}^{M} \sum_{k^{\prime} \in \mathbb{Z}^{d}} r_{m}\left(k-k^{\prime}\right) y_{m}\left(k^{\prime}\right), k \in \mathbb{Z}^{d}$. From the construction of vectors $\left(r_{m}(k)\right)_{k \in \mathbb{Z}^{d}}$, we see that $c(k)=\sum_{m=1}^{M} \sum_{k^{\prime} \in \mathbb{Z}^{d}} r_{m}(k-$ $\left.k^{\prime}\right)\left\langle\tilde{h}, \psi_{m}\left(\cdot-k^{\prime}\right)\right\rangle$ for all $k \in \mathbb{Z}^{d}$, where $\tilde{h}=\sum_{k \in \mathbb{Z}^{d}} c(k) \varphi(\cdot-k)$. This together with (8.13) gives that

$$
\|\tilde{c}-c\|_{\infty} \leq D_{2}\left(\sum_{m=1}^{M}\left\|\psi_{m}\right\|_{1}\right)\|c\|_{\infty}\left\|\sum_{k \in \mathbb{Z}^{d}}|\varphi(\cdot-k-\sigma(k))-\varphi(\cdot-k)|\right\|_{\infty} \leq C_{0}\|c\|_{\infty}\|\sigma\|_{\infty}
$$

for some positive constant $C_{0}$. Therefore, if $\delta_{1} \leq 1 /\left(3 C_{0} L^{2}\right)$, the vector $\tilde{c}_{0}:=$ $\left(\tilde{c}_{0}(k)\right)_{k \in \mathbb{Z}^{d}}$ defined by

$$
\tilde{c}_{0}(k)= \begin{cases}\tilde{c}(k) & \text { if }|\tilde{c}(k)| \geq 1 /(2 L) \\ 0 & \text { otherwise }\end{cases}
$$

has the same support, say $\Lambda_{0}$, as the one of the amplitude vector $c$ of the signal $h$ to be recovered, and satisfies

$$
\left\|\tilde{c}_{0}-c\right\|_{\infty} \leq C_{0} L\|\sigma\|_{\infty}
$$

So we may consider $\sum_{k \in \Lambda_{0}} \tilde{c}_{0}(k) \varphi(\cdot-k)$ as an approximation of the signal $h=$ $\sum_{k \in \Lambda_{0}} c(k) \varphi(\cdot-k-\sigma(k))=\sum_{k \in \mathbb{Z}^{d}} c(k) \varphi(\cdot-k-\sigma(k))$ to be recovered. 
Let $c_{0}=\left(\tilde{c}_{0}(\lambda)\right)_{\lambda \in \Lambda_{0}}, \Psi=\left\{\psi_{m}(\cdot-k)\right\}_{1 \leq m \leq M, k \in \mathbb{Z}^{d}}$, and $S_{\Lambda_{0}, c_{0}}$ be the the linearization matrix in (1.17). Then for any $e=(e(\lambda))_{\lambda \in \Lambda_{0}} \in\left(\ell^{2}\left(\Lambda_{0}\right)\right)^{d+1}$,

$$
\begin{aligned}
\left\|S_{\Lambda_{0}, c_{0}} e\right\|_{2}^{2} & =\sum_{m=1}^{M} \sum_{k \in \mathbb{Z}^{d}}\left|\left\langle\psi_{m}(\cdot-k), \sum_{\lambda \in \Lambda_{0}}\left(-\tilde{c}_{0}(\lambda) e_{g}(\lambda)^{T} \nabla \varphi(\cdot-\lambda)+e_{o}(\lambda) \varphi(\cdot-\lambda)\right)\right\rangle\right|^{2} \\
& \geq C\left(\sum_{\lambda \in \Lambda_{0}}\left|\tilde{c}_{0}(\lambda)\right|^{2}\left|e_{g}(\lambda)\right|^{2}+\left|e_{o}(\lambda)\right|^{2}\right) \geq C L^{-2}\|e\|_{\left(\ell^{2}\left(\Lambda_{0}\right)\right)^{d+1}}^{2}
\end{aligned}
$$

and

$$
\begin{aligned}
\left\|S_{\Lambda_{0}, c_{0}} e\right\|_{2}^{2} & \leq C\left\|\sum_{\lambda \in \Lambda_{0}}\left(-\tilde{c}_{0}(\lambda) e_{g}(\lambda)^{T} \nabla \varphi(\cdot-\lambda)+e_{o}(\lambda) \varphi(\cdot-\lambda)\right)\right\|_{2}^{2} \\
& \leq C\|e\|_{\left(\ell^{2}\left(\Lambda_{0}\right)\right)^{d+1}}^{2}\left(1+\left\|\tilde{c}_{0}\right\|_{\ell^{\infty}\left(\Lambda_{0}\right)}\right)^{2} \leq C L^{2}\|e\|_{\left(\ell^{2}\left(\Lambda_{0}\right)\right)^{d+1}}^{2}
\end{aligned}
$$

by (1.21), (18.1), (8.13) and (18.18), where $e(\lambda)=\left(\begin{array}{l}e_{g}(\lambda) \\ e_{o}(\lambda)\end{array}\right), \lambda \in \Lambda_{0}$. The above estimates lead to the conclusion that the linearization matrix $S_{\Lambda_{0}, c_{0}}$ satisfies the stability condition (1.16). This together with (8.18) and Theorem 8.1 completes the proof.

8.3. Numerical simulation. In the following simulation, the signal $h$ to be recovered has the following parametric representation

$$
h(t)=\sum_{i=1}^{20} a_{i} \varphi_{0}\left(t-t_{i}\right),
$$

where $\varphi_{0}(t)=\exp \left(-4(\pi / 2)^{2 / 3} t^{2}\right)$, the innovation positions $t_{i}, 1 \leq i \leq 20$, are randomly selected numbers in $[0.5,19.5]$ satisfying $t_{1}=0.5, t_{20}=19.5$ and $0.5 \leq$ $\min _{1 \leq i \leq 19} t_{i+1}-t_{i} \leq \max _{1 \leq i \leq 19} t_{i+1}-t_{i} \leq 1.5$, and the amplitudes $a_{i}, 1 \leq i \leq 20$, are random selected numbers in $[-1,-0.1] \cap[0.1,1]$. We consider the recovery of such a signal $h$ as it can be used to model diffusion of point sources of pollution and the generating signal $\varphi_{0}$ does not satisfy the Strang-Fix condition.

The given samples $y_{j}, 1 \leq j \leq 41$, of the signal $h$ are obtained by average sampling the companded signal $\sin (\pi h(t) / 2)$ by the shifted square-root sampler $\psi_{0}$,

$$
y_{j}=\left\langle\sin (\pi h(\cdot) / 2) \chi_{[0,20]}, \psi_{0}(\cdot+1-j / 2)\right\rangle, \quad 1 \leq j \leq 41,
$$

where

$$
\psi_{0}(t)= \begin{cases}\max (1 / 4-|2 t-1 / 4|, 0) & \text { if } 0 \leq t \leq 1 / 4 \\ 4 t-1 & \text { if } 1 / 4 \leq t \leq 1 / 2 \\ 1 & \text { if } 1 / 2 \leq t \leq 1 \\ 0 & \text { otherwise }\end{cases}
$$

is the square-root sampling kernel, see Figure 4. The approximate innovation positions $t_{0, i}$ and amplitudes $c_{0, i}, 1 \leq i \leq 20$, are given by

$$
a_{0, i}=\left\lfloor 10 a_{i}\right\rfloor / 10 \text { and } t_{0, i}=\left\lfloor 10 t_{i}\right\rfloor / 10,1 \leq i \leq 20 \text {, }
$$

where $\lfloor a\rfloor$ is the largest integer less than or equal to $a \in \mathbb{R}$. We use the VanCittert iteration (8.12) in Theorem 8.2 to recover the exact innovation positions 


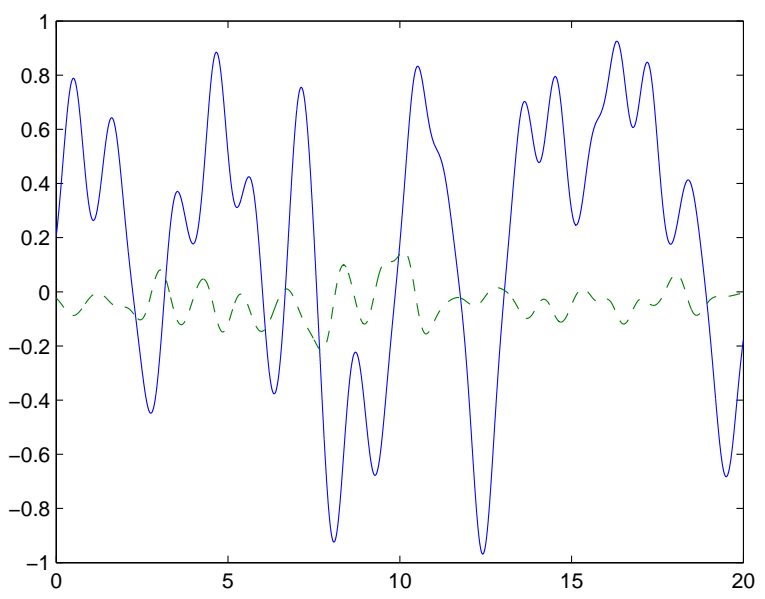

FIGURE 3. The original signal $h(t)=\sum_{i=1}^{20} a_{i} \varphi_{0}\left(t-t_{i}\right)$ to be recovered is plotted in a continuous line, while the difference between the original signal $h(t)$ and the approximate signal $h_{\text {app }}(t)=\sum_{i=1}^{20} a_{0, i} \varphi_{0}\left(t-t_{0, i}\right)$ is plotted in a dashed line.

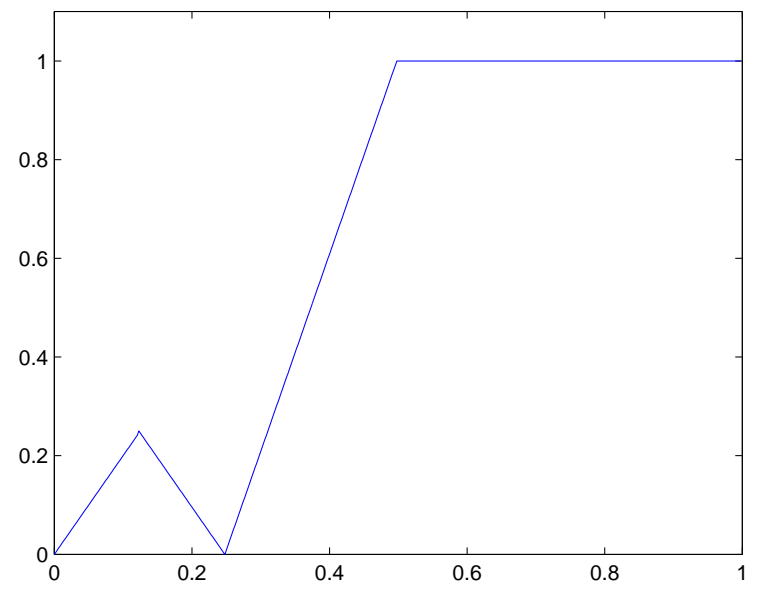

FiguRE 4. The square-root sample $\psi_{0}$ in (8.21).

and amplitudes with $c_{0}=\sigma_{0}=0$ as initials and $\alpha=0.5$ as the relaxation factor. In Tables 3 below, we list the amplitude errors $\left\|c_{n}-c_{\infty}\right\|_{\infty}$ in the second column, the innovation position errors $\left\|\sigma_{n}-\sigma_{\infty}\right\|_{\infty}$ in the third column, and the sampling errors $\left\|\left\langle F\left(h_{n}\right)-F(h), \Psi\right\rangle\right\|_{\infty}$ in the forth column, where $F(t)=\sin (\pi t / 2)$ and $\Psi=\left(\psi_{0}(\cdot+1-j / 2) \chi_{[0,20]}\right)_{1 \leq j \leq 41}$. We observe that the sequences $\sigma_{n}$ and $c_{n}, n \geq 0$, in the Van-Cittert iteration (8.12) converges and their limits, denoted by $\sigma_{\infty}$ and $c_{\infty}$ respectively, satisfy $\sigma_{\infty}=\left(t_{i}-t_{0, i}\right)_{1 \leq i \leq 20}$ and $c_{\infty}=\left(a_{i}-a_{0, i}\right)_{1 \leq i \leq 20}$. Hence we reach to the desired conclusion that the signal $h$ in (8.19) can be recovered accurately 
TABLE 3. Local identification of innovation positions and qualification of amplitudes via the Van-Cittert iteration method

\begin{tabular}{cccc}
\hline \hline $\mathrm{n}$ & $\left\|c_{n}-c_{\infty}\right\|_{\infty}$ & $\left\|\sigma_{n}-\sigma_{\infty}\right\|_{\infty}$ & $\left\|\left\langle F\left(h_{n}\right)-F(h), \Psi\right\rangle\right\|_{\infty}$ \\
\hline 1 & 0.1465 & 0.4085 & 0.2805 \\
5 & 0.1459 & 0.0292 & 0.1590 \\
10 & 0.0088 & 0.0045 & 0.0140 \\
15 & 0.0005 & 0.0004 & 0.0007 \\
$\geq 20$ & 0.0000 & 0.0000 & 0.0000 \\
\hline \hline
\end{tabular}

from its samples via the Van-Cittert iteration (8.12) when its innovation positions and amplitudes are given approximately. Moreover, the above recovery process is stable in the presence of bounded noise in Theorem 5.1. In Table 4 below, we list numerical results when we apply the Van-Citert iteration (8.12) with exact samples $y_{j}, 1 \leq j \leq 41$, in (8.20) replaced by the samples $\tilde{y}_{j}, 1 \leq j \leq 41$, corrupted by $5 \%$ random additive noise, i.e.,

$$
\tilde{y}_{j}=y_{j}+0.05 * \epsilon_{j} *\left(\max _{1 \leq k \leq 41}\left|y_{k}\right|\right), 1 \leq j \leq 41,
$$

where $\epsilon_{j} \in[-1,1], 1 \leq j \leq 41$, are random noise.

TABLE 4. Local identification of innovation positions and qualification of amplitudes via the Van-Cittert iteration method in the presence of $5 \%$ bounded random noise

\begin{tabular}{cccc}
\hline \hline $\mathrm{n}$ & $\left\|c_{n}-c_{\infty}\right\|_{\infty}$ & $\left\|\sigma_{n}-\sigma_{\infty}\right\|_{\infty}$ & $\left\|\left\langle F\left(h_{n}\right)-F(h), \Psi\right\rangle\right\|_{\infty}$ \\
\hline 1 & 0.1725 & 0.4593 & 0.2805 \\
5 & 0.3059 & 0.0818 & 0.3769 \\
10 & 0.1110 & 0.0716 & 0.0820 \\
15 & 0.1110 & 0.0847 & 0.0648 \\
$\geq 19$ & 0.1110 & 0.0853 & 0.0648 \\
\hline \hline
\end{tabular}

Acknowledgement The author would like to thank anonymous reviewers for their valuable comments for the improvement of the manuscript.

\section{REFERENCES}

[1] A. Aldroubi, A. Baskakov and I. Krishtal, Slanted matrices, Banach frames, and sampling, J. Funct. Anal., 255(2008), 1667-1691.

[2] A. Aldroubi and K. Gröchenig, Nonuniform sampling and reconstruction in shift-invariant space, SIAM Review, 43(2001), 585-620.

[3] A. Aldroubi, Q. Sun, and W.-S. Tang, Convolution, average sampling and a Calderon resolution of the identity for shift-invariant spaces, J. Fourier Anal. Appl., 11(2005), 215-244.

[4] R. Balan, P. G. Casazza, C. Heil, and Z. Landau, Density, overcompleteness and localization of frames I. Theory; II. Gabor system, J. Fourier Anal. Appl., 12(2006), pp. 105-143 and pp. 309-344.

[5] R. Balan, The noncommutative Wiener lemma, linear independence, and special properties of the algebra of time-frequency shift operators, Trans. Amer. Math. Soc., 360(2008), 39213941. 
[6] A. G. Baskakov, Wiener's theorem and asymptotic estimates for elements of inverse matrices, Funktsional. Anal. i Prilozhen, 24(1990), 64-65; translation in Funct. Anal. Appl., 24(1990), $222-224$.

[7] N. Bi, M. Z. Nashed and Q. Sun, Reconstructing signals with finite rate of innovation from noisy samples, Acta Appl. Math., 107(2009), 339-372.

[8] B. Blackadar and J. Cuntz. Differential Banach algebra norms and smooth subalgebras of $C^{*}$-algebras, J. Operator Theory, 26(1991), 255-282.

[9] F. E. Browder, Nonlinear mappings of nonexpansive and accretive type in Banach spaces, Bull. Amer. Math. Soc., 73(1967), 875-882.

[10] O. Christensen and T. Strohmer, The finite section method and problems in frame theory, J. Approx. Theory, 133(2005), 221-237.

[11] K. R. Coombes, J. M. Koomen, K. A. Baggerly, J. S. Morris, and R. Kobayashi, Understanding the characteristics of mass spectrometry data through the use of simulation, Cancer Informatics, 1(2005), 41-52.

[12] R. J.-M. Cramer, R. A. Scholtz, and M. Z. Win, Evaluation of an ultra wide-band propagation channel, IEEE Trans. Antennas and Propagation, 50(2002), 561-569.

[13] S. Dahlke, M. Fornasier, and K. Gröchenig, Optimal adaptive computations in the Jaffard algebra and localized frames, J. Approx. Th., 162(2010), 153-185.

[14] R. A. DeVore and G. G. Lorentz, Constructive Approximation, Springer-Verlag, Berlin, 1993.

[15] D. Donoho, Compressive sampling, IEEE Trans. Inform. Theory, 52(2006), 1289-1306.

[16] P. L. Dragotti, M. Vetterli and T. Blu, Sampling moments and reconstructing signals of finite rate of innovation: Shannon meets Strang-Fix, IEEE Trans. Signal Processing, 55(2007), $1741-1757$.

[17] T. G. Dvorkind, Y. C. Eldar and E. Matusiak, Nonlinear and nonideal sampling: Theory and Methods, IEEE Trans. Signal Process., 56(2008), 5874-5890.

[18] H. Farid, Blind inverse gamma correction, IEEE Trans. Image Process., 10(2001), 1428-1433.

[19] T. Faktor, T. Michaeli, and Y. C. Eldar, Nonlinear and nonideal sampling revisited, IEEE Signal Processing Letter, 17(2010), 205-208.

[20] I. Gohberg, M. A. Kaashoek, and H. J. Woerdeman, The band method for positive and strictly contractive extension problems: an alternative version and new applications, Integral Equ. Oper. Theory, 12(1989), 343-382.

[21] K. Gröchenig, Wiener's lemma: theme and variations, an introduction to spectral invariance and its applications, In Four Short Courses on Harmonic Analysis: Wavelets, Frames, TimeFrequency Methods, and Applications to Signal and Image Analysis, edited by P. Massopust and B. Forster, Birkhäuser, Boston, 2010.

[22] K. Gröchenig, Time-frequency analysis of Sjöstrands class, Rev. Mat. Iberoam., 22(2006), 703-724.

[23] K. Gröchenig and A. Klotz, Noncommutative approximation: inverse-closed subalgebras and off-diagonal decay of matrices, Constr. Approx., 32(2010), 429-466.

[24] K. Gröchenig and A. Klotz, Norm-controlled inversion in smooth Banach algebras I, J. London Math. Soc., to appear.

[25] K. Gröchenig and M. Leinert, Wiener's lemma for twisted convolution and Gabor frames, $J$. Amer. Math. Soc., 17(2003), 1-18.

[26] K. Gröchenig and M. Leinert, Symmetry of matrix algebras and symbolic calculus for infinite matrices, Trans, Amer. Math. Soc., 358(2006), 2695-2711.

[27] K. Gröchenig and Z. Rzeszotnik, Almost diagonlization of pseudodifferntial operators, Ann. Inst. Fourier, 58(2008), 2279-2314.

[28] K. Gröchenig, Z. Rzeszotnik, and T. Strohmer, Convergence analysis of the finite section method and Banach algebras of matrices, Integral Equ. Oper. Theory, 67(2010), 183-202.

[29] K. Gröchenig, and T. Strohmer, Pseudodifferential operators on locally compact Abelian groups and Sjöstrands symbol class, J. Reine Angew. Math., 613(2007), 121-146. 
[30] P. Hall and J. Jin, Innovated higher criticism for detecting sparse signals in correlated noise Ann. Statist., 38(2010), 1686-1732.

[31] G. C. Holst, CCD Arrays Camera and Displays, Bellingham, WA, SPIE Press, 1996.

[32] S. Jaffard, Properiétés des matrices bien localisées prés de leur diagonale et quelques applications, Ann. Inst. Henri Poincaré, 7(1990), 461-476.

[33] T. Kato, Nonlinear semigroups and evolution equations, J. Math. Soc. Japan, 19(1967), 508-520.

[34] S. Kawai, M. Marimoto, N. Mutoh, and N. Teranishi, Photo response analysis in CCD image sensors with a VOD structure, IEEE Trans. Electron Devices, 42(1995), 652-655.

[35] E. Kissin and V. S. Shulman. Differential properties of some dense subalgebras of $C^{*}$-algebras, Proc. Edinburgh Math. Soc. 37(1994), 399-422.

[36] K. Kose, K. Endoh, and T. Inouye, Nonlinear amplitude compression in magnetic resonance imaging: Quantization noise reduction and data memory saving, IEEE Aerosp. Electron. Syst,. Mag., 5(1990), 27-30.

[37] I. Krishtal, Wiener's lemma: pictures at exhibition, Revista Union Matematica Argentina, 52(2011), 61-79.

[38] I. Krishtal, and K. A. Okoudjou, Invertibility of the Gabor frame operator on the Wiener amalgam space, J. Approx. Theory, 153(2008), 212-224.

[39] J. Kusuma, I. Maravic and M. Vetterli, Sampling with finite rate of innovation: channel and timing estimatation for UWB and GPS, IEEE Conference on Communication 2003, Achorage, AK.

[40] H. J. Landau and W. L. Miranker, The recovery of distorted band-limited signals, J. Math. Anal. Appl., 2(1961), 97-104.

[41] Y. M. Lu and M. N. Do, A theory for sampling signals from a union of subspaces, IEEE Transactions on Signal Processing, 56(2008), 2334-2345.

[42] I. Maravic and M. Vetterli, Sampling and reconstruction of signals with finite rate of innovation in the presence of noise, IEEE Trans. Signal Processing, 53(2005), 2788-2805.

[43] P. Marziliano, M. Vetterli, and T. Blu, Sampling and exact reconstruction of bandlimited signals with shot noise, IEEE Trans. Inform. Theory, 52(2006), 2230-2233.

[44] J. L. Mannos and D. J. Sakrison, The effects of a visual fidelity criterion on the encoding of images, IEEE Trans. Inform. Theory, 20(1974), 525-536.

[45] T. Michaeli and Y. C. Eldar, Xampling at the rate of innovation, IEEE Trans. Signal Processing, 60(2012), 1121-1133.

[46] G. J. Minty, Monotone (nonlinear) operators in Hilbert space, Duke Math. J., 29(1962), $341-346$.

[47] N. Motee and A. Jadbabaie, Optimal control of spatially distributed systems, IEEE Trans. Automatic Control, 53(7)(2008), 1616-1629.

[48] N. Motee and A. Jadbabaie, Distributed multi-parametric quadratic programming, IEEE Trans. Automatic Control, 54(10)(2009), 2279-2289.

[49] M. Z. Nashed and Q. Sun, Sampling and reconstruction of signals in a reproducing kernel subspace of $L^{p}\left(\mathbb{R}^{d}\right)$, J. Funct. Anal., 258(2010), 2422-2452.

[50] N. Nikolski, In search of the invisible spectrum, Ann. Inst. Fourier (Grenoble), 49(1999), 1925-1998.

[51] F. Pejovic and D. Maksimovic, A new algorithm for simulation of power electronic systems using piecewise-linear device models, IEEE Trans. Power Electron., 10(1995), 340-348.

[52] M. A. Rieffel, Leibniz seminorms for "matrix algebras converge to the sphere". In Quanta of maths, volume 11 of Clay Math. Proc., Amer. Math. Soc., Providence, RI, 2010. pages $543-578$.

[53] I. W. Sandberg, Notes on PQ theorems, IEEE Trans. Circuit and Systems-I: Fundamental Theory and Applications, 41(1994), 303-307.

[54] K. Shafique and M. Shah, Estimation of radiometric response function of a color camera from differently illuminated images, In Proc. Int. Conf. Image Process., 2004, 2339-2342. 
[55] C. E. Shin and Q. Sun, Stability of localized operators, J. Funct. Anal., 256(2009), 24172439.

[56] P. Shukla and P. L. Dragotti, Sampling schemes for multidimensional signals with finite rate of innovation, IEEE Trans. Signal Processing, 55(2007), 3670-3686.

[57] J. Sjöstrand, Wiener type algebra of pseudodifferential operators, Centre de Mathematiques, Ecole Polytechnique, Palaiseau France, Seminaire 1994-1995, December 1994.

[58] Q. Sun, Wiener's lemma for infinite matrices with polynomial off-diagonal decay, C. Acad. Sci. Paris Ser I, 340(2005), 567-570.

[59] Q. Sun, Non-uniform sampling and reconstruction for signals with finite rate of innovations, SIAM J. Math. Anal., 38(2006), 1389-1422.

[60] Q. Sun, Wiener's lemma for infinite matrices, Trans. Amer. Math. Soc., 359(2007), 30993123 .

[61] Q. Sun, Frames in spaces with finite rate of innovations, Adv. Comput. Math., 28(2008), 301-329.

[62] Q. Sun, Local reconstruction for sampling in shift-invariant spaces, Adv. Comput. Math., 32(2010), 335-352.

[63] Q. Sun, Wiener's lemma for infinite matrices II, Constr. Approx., 34(2011), 209-235.

[64] T. Tao, A quantitative proof of Wiener's theorem, http://www.math.ucla.edu/ tao/preprints/harmonic.html, 2005

[65] M. Unser, Sampling - 50 years after Shannon, Proceedings of the IEEE, 88(2000), 569-587.

[66] M. Vetterli, P. Marziliano, and T. Blu, Sampling signals with finite rate of innovation, IEEE Trans. Signal Proc., 50(2002), 1417-1428.

[67] Z. Wang and A. C. Bovik, Mean squared error: love it or leave it?- A new look at signal fidelity measures, IEEE Signal Processing Magazine, 98(2009), 98-117.

[68] N. Wiener, Tauberian Theorem, Ann. Math., 33(1932), 1-100.

[69] E. Zeidler, Nonlinear Functional Analysis and its Applications, Vol. 1-5, Springer-Verlag.

Sun: Department of Mathematics, University of Central Florida, Orlando, FL 32816

E-mail address: qiyu.sun@ucf.edu 